\title{
Anabases
}

ANABASES Traditions et réceptions de l'Antiquité

11 | 2010

Varia

\section{Hubertus Goltzius e la Civitas almae urbis Romae}

\section{Maria Luisa Napolitano}

\section{OpenEdition}

\section{Journals}

Edizione digitale

URL: http://journals.openedition.org/anabases/769

DOI: 10.4000/anabases.769

ISSN: 2256-9421

\section{Editore}

E.R.A.S.M.E.

\section{Edizione cartacea}

Data di pubblicazione: 1 marzo 2010

Paginazione: 55-94

ISSN: 1774-4296

Notizia bibliografica digitale

Maria Luisa Napolitano, « Hubertus Goltzius e la Civitas almae urbis Romae », Anabases [Online], 11]

2010, Messo online il 01 mars 2013, consultato il 20 octobre 2019. URL : http://

journals.openedition.org/anabases/769 ; DOI : 10.4000/anabases.769

(c) Anabases 


\section{Hubertus Goltzius}

e la Civitas almae urbis Romae

Maria Luisa Napolitano

Il ritratto, l'iscrizione, il personaggio

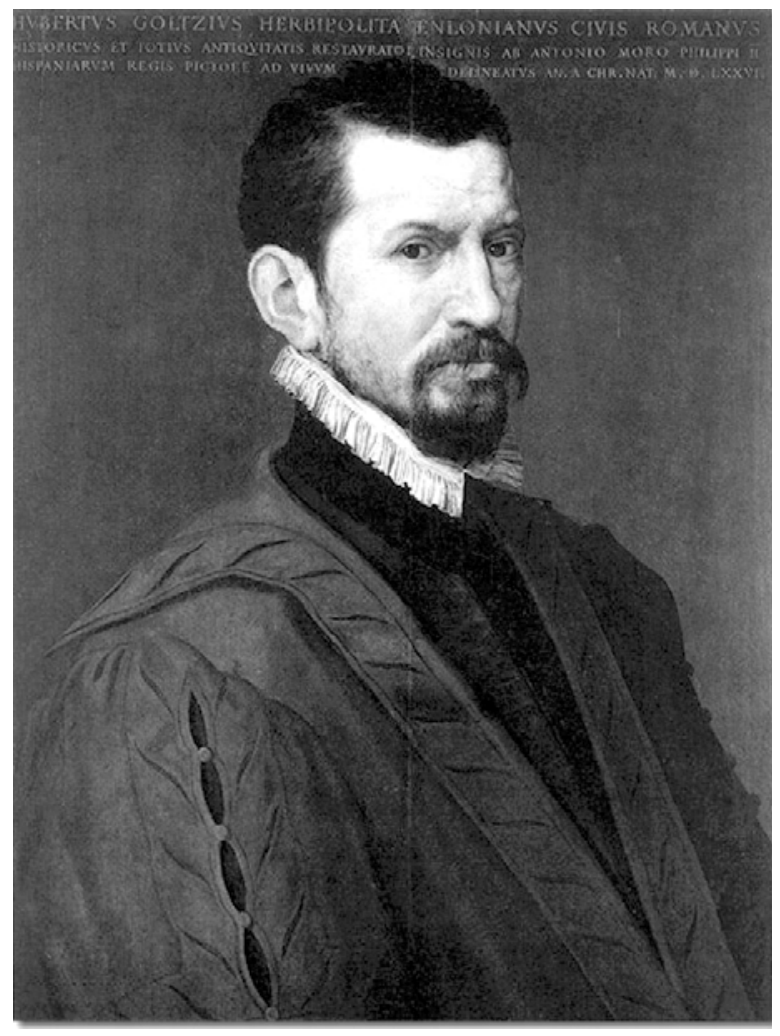


L'ultimo dipinto di Antonio Moro ${ }^{1}$ ritrattista ufficiale alla corte di Spagna ${ }^{2}$ e celebre pittore nell'Europa del Rinascimento ${ }^{3}$, è il ritratto di Hubertus Goltzius di Venloo, oggi al Museum of Fine Arts of Belgium, a Bruxelles. Nelle sue Vite degli illustri pittori fiamminghi, olandesi e tedeschi, del 1604, il van Mander ambienta in un clima di sodale familiarità tra i due l'occasione dell'esecuzione del quadro ${ }^{4}$, sul quale, sovradipinta in

1 Antoon, Antonis o Anthonius, Mor o Mor van Dashorst: Utrecht 1516/20 - Anversa 1576.

2 Accolto presso la corte di Spagna per il tramite del cardinale di Granvela, Antonio Moro diviene soprattutto celebre come ritrattista dei potenti. Dal 1552 lavora per Carlo V, quindi per Filippo II; trasferitosi poi a Bruxelles, collabora con il Duca d'Alba, ottenendone i favori. Per il confronto con i modelli artistici italiani, in particolare Tiziano, Michelangelo, Raffaello, vd. ora J. Woodall, Anthonis Mor: Art and Authority, Zwolle, 2008, p. 20-25, 40, 96, 109-111, 131, 170 ss., 188, 209-212, passim; per il modello rappresentato da Roma antiqua vd. ibid., p. 95-133, passim. Sulle relazioni di A. Moro con gli ambienti di corte e di potere europei del XVI secolo vd. tra gli altri: K. VAN MANDER, Le vite degli illustri pittori fiamminghi, olandesi e tedeschi, a cura di R. de Mambro Santos, Roma, 2000 [1604], p. 193-195; F. DE BONI (a cura di), Biografia degli artisti, Venezia, 1840, p. 681-2; H. Hymans, Antonio Moro. Son oeuvre et son temps, Brussels, 1910; L. Cust, "Notes on Pictures in the Royal Collections-XVIII. On Some Portraits Attributed to Antonio Moro and on a Life of the Painter by Henri Hymans", The Burlington Magazine Publications 91 (1910), p. 5-12.; D. CATTON Rich, "A Portrait by Antonio Moro", Bulletin of the Art Institute of Chicago (1907-1951) 26 (1932), p. 13-15, part. 14; A. SEBASTIANI (a cura di), I Fiamminghi e l'Italia, Esposizione Bruges-Venezia-Roma 1951, Venezia, 1951, p. 42; Collection of Mediaeval and Renaissance Paintings, Fogg Art Museum, Harvard, 2004, p. 308 e passim; E. Du Gue Trapier, Catalogue of Paintings $\left(16^{\text {th }}, 17^{\text {th }}\right.$ and $\left.18^{\text {th }}\right)$, extracted from: Hispanic Notes \& Monographs Essays, Studies and Brief Biographies Issued by the Hispanic Society of America Catalogue of Paintings in the Collection of the Hispanic Society of America, New York, 2007, p. 15-30 e passim; Woodall, Anthonis Mor, p. 18-22, 26, 135-138, 184 ss., 339, passim.

3 Lusinghiero il giudizio del Vasari: «[Antonio Moro] di Utrec in Olanda, pittore del Re Cattolico, del quale nel ritrarre ciò che vuole di naturale dicono contendere con la natura, e ingannare gli occhi benissimo. Scrivemi il detto Lampsonio che il Moro, il quale è di gentilissimi costumi, ha fatto una tavola bellissima d'un Cristo che risuscita con due angeli, e San Piero e San Paolo, che è una cosa meravigliosa [...]» (G. VASARI, Vite de' più eccellenti pittori scultori e architetti, a cura di G. Della Valle, Siena, 1794, p. 71-72).

4 «Hubertus [...] fu tenuto in alta considerazione presso dotti e sovrani; regalò ad Antonio Moro uno dei suoi pregiati libri di medaglie. L'artista, felice di ricevere un dono così prezioso, disse all'amico di volerlo ringraziare con la sua arte: lo pregò, pertanto, di recarsi a casa sua nelle due mattine seguenti, affinché potesse realizzare il suo ritratto. In tali occasioni, dopo aver offerto una colazione assai abbondante, Moro si congedava dallo scrittore senza aver dipinto nulla. La terza mattinata, infine, eseguì il ritratto dell'erudito in meno di un'ora, ovvero entro un brevissimo spazio di tempo, ottenendo un'immagine stupefacente per quanto riguarda la somiglianza e la resa, poiché nei giorni precedenti egli era riuscito a imprimere nella sua mente la vera essenza dell'umanista. Questo ritratto si trova tuttora a Bruges presso la vedova o i parenti, e venne inciso anche all'interno di uno dei suoi volumi» (VAN MANDER, Le vite degli illustri pittori, p. 239). 
alto, è leggibile una iscrizione datata $1576^{5}$, che ha contribuito alla sicura identificazione del personaggio rappresentato. Essa così recita:

\section{HUBERTUS GOLTZIUS HERBIPOLITA VENLONIANUS CIVIS ROMANUS}

HISTORICUS ET TOTIUS ANTIQUITATIS RESTAURATOR INSIGNIS AB ANTONIO MORO PHILIPPI II HISPANIARUM REGIS PICTORE AD VIVUM DELINEATUS AN. A CHR. NAT. M.D. LXXVI.

L'espressione HISTORICUS ET TOTIUS ANTIQUITATIS RESTAURATOR INSIGNIS EVOca l'attività di Hubertus Goltzius ${ }^{6}$ come historicus, autore di ricostruzioni di aspetti del mondo antico, e come restaurator insignis, distintosi nel recupero e nella trasmissione di antichità all'età presente. Per inciso, la voce insignis evoca la semantica materiale del signum come "marchio», "contrassegno», «incisione»; ed è Goltzius stesso a predisporre le incisioni delle proprie opere, incluse le Tavole monetali. La qualifica di CIVIS ROMANUS è invece termine tecnico specifico e implica l'ammissione effettiva di Goltzius alla Civitas Romana, la sua ufficiale iscrizione nei registri municipali dei cives di Roma. Nato e vissuto nelle Fiandre, tra la natale Venloo e i poli di Liegi, Anversa, Bruges, Hubertus Goltzius (1526-1583) risulta molto noto agli ambienti di cultura e di potere dell'Europa del Cinquecento come eccellente pittore, incisore, umanista, storico, numismatico, editore, mercante d'arte (attività quest'ultima intrapresa negli anni di Anversa e dell'incontro con il geografo e amico Ortelius, dal 1546 in poi). Deve però la maggiore notorietà alle sue opere, nelle quali la documentazione numismatica (con

5 L'iscrizione è aggiunta al dipinto, datato al 1574 sulla base di una lettera ad Abraham Ortelius del 21 febbraio 1574 in cui si menziona il quadro (J.H. Hessels [edidit], Epistulae Ortelianae. Abrahami Ortelii [Geographi Antverpiensis] et virorum eruditorum ad eundem et ad Jacobum Colium Ortelianum [Abrahami Ortelii sororis filium] Epistulae, cum aliquot aliis epistulis et tractatibus quibusdam ab utroque collectis [1524-1628], ex autographis mandante Ecclesia Londino-Batava, 1887; reproductio phototypica: O. ZeLler, Osnabrück, 1969, p. 109).

6 Su Hubertus Goltzius (Venloo 1526 - Bruges 1583) e in particolare sulla sua nozione di Magna Graecia è in corso una ricerca di dottorato di chi scrive per l'Università degli Studi di Napoli «Federico II». Sull'opera goltziana i contributi più accurati e accessibili sono certamente quelli di C.E. Dekesel, che ha studiato tale produzione editoriale con particolare riguardo alla prima pubblicazione del 1557, Imagines imperatorum (C.E. DeKesel, Hubertus Goltzius the Father of Ancient Numismatics. An Annoted and Illustrated Bibliography, Gandavum Flandrorum, 1988; ID., Bibliotheca Nummaria. Bibliography of $16^{\text {th }}$ Century Numismatic Books, London, 1997; ID., "Hubertus Goltzius [Venloo 1526 - Bruges 1583] and his Icones Imperatorum Romanorum", in R. PERA [a cura di], L'immaginario del potere. Studi di iconografia monetale, Roma, 2005, p. 259-277). Le qualifiche indicate da Antonio Moro nell'iscrizione implicano una selezione tra le molteplici attività del Goltzius, per indicazione forse dello stesso studioso. Non v'è cenno all'attività artistica, di pittore e di incisore, agli interessi antiquari e al collezionismo, mentre resta nell'ombra il lavoro della officina editoriale, forse da intendersi incluso nella espressione totius antiquitatis restaurator. 
l'epigrafia, sia pure in misura minore) funge da agente documentale predominante. Egli così risponde per un verso alle richieste degli ambienti intellettuali del momento, caratterizzati dall'appassionato collezionismo di oggetti particolari e materiali antichi, in particolare di monete e medaglie greche e romane, ma nel contempo trascendeva l'invalsa prospettiva corrente di raccolta e accumulo, con risvolti di prestigio sociale oltre che economici spesso non trascurabili.

Parallelamente, nell'Europa del secolo XvI si sviluppa tutto un filone letterario dal carattere che oggi definiremmo erudito, ma che per l'epoca rappresentava una tipologia di umanesimo metodologicamente fondata su criteri di scientificità, un aspetto dell'antiquaria ${ }^{7}$. Si afferma l'esigenza di ordinare, classificare, riprodurre in forma sistematica i materiali antichi emersi da rinvenimenti fortuiti, o per così dire archeologici, e da acquisti e scambi tra privati, esibiti nelle collezioni personali e familiari a memoria imperitura ${ }^{8}$. L'esame della documentazione numismatica, in particolare dell'iconografia monetale, si carica di valenze simboliche nella sua rappresentazione immaginifica del passato avvertito come sede ideale di valori vivi, e nella sua funzione di tramite tra antichità e presente, impulso anche per la letteratura emblematica propria di questa fase.

In un primo lungo periodo, dal 1557 al 1574, Goltzius si dedica appassionatamente allo studio del mondo romano, per la cui ricostruzione affianca alla guida dei classici greci e latini, riletti, parafrasati, illustrati, il dato numismatico riconosciuto e valorizzato come elemento di cultura materiale. Nel 1557, prima di lasciare Anversa, apprezzato nei maggiori ambienti artistici e culturali della città, divenuto amico di Abraham Ortelius, egli pubblica la prima sua opera, Imperatorum imagines ${ }^{9}$, in latino e nelle maggiori lingue europee, cui deve la grande notorietà in Europa e in Italia ${ }^{10}$. In

7 La definizione cinquecentesca di antiquaria è ricostruita in A. Momigliano, "Ancient History and the Antiquarian", Journal of the Warburg and Courtauld Institutes 13 (1950), p. 285-315: più del semplice storico, l'antiquario collazionava e studiava le testimonianze antiche nel loro complesso, la documentazione letteraria, quella epigrafico-numismatica e i reperti materiali in genere, in vista di una ricostruzione esaustiva dell'antico che oggi definiremmo storica.

8 Per la valorizzazione del documento numismatico nel complesso della produzione editoriale europea del XVI secolo sull'antichità, soprattutto romana, vd. in particolare e tra gli altri F. BASSOLI, Monete e medaglie nel libro antico dal XV al XIX secolo, Firenze, 1985, p. 7-17; J. Cunnally, Images of the Illustrious. The Numismatic Presence in the Renaissance, Princeton, 1999, p. 3-9, 34-37.

9 Vivae omnium fere imperatorum imagines, a C. Iulio Caesare usque ad Carolum 5. et Ferdinandum eius fratrem, ex antiquis veterum numismatis solertissime, non vt olim ab aliis, sed vere ac fideliter adumbratae, nec non eorundem vitae, acta, mores, virtute, vitia, suis coloribus historico penicillo delineatae. [...] per Hubertum Goltzium Wirtzburgensem pictorem, Antuerpiae, 1557 (Excus. Antuerpiae: cura \& aere Huberti Goltzii, in officina Aegidij Copenij Diesthemij).

10 Per i cui lettori fa predisporre due versioni, in toscano e in italiano, oltre al testo in latino che ha destinazione «universale». 
essa con originale sistema di incisione e rappresentazione ${ }^{11}$ riproduce in icone monetali, con breve motto a commento, i ritratti degli imperatori da Roma antica all'epoca contemporanea. Del 1563 è il C. Iulius Caesar ${ }^{12}$, primo di una serie di volumi che si prevedevano incentrati sulla storia di Roma da Cesare in poi, pubblicato a Bruges per i tipi della officina editoriale Goltziana, diretta da Goltzius stesso e patrocinata dal mecenate Marc Laurin, signore di Watervliet. Nel 1566 Goltzius pubblica i Fasti ${ }^{13}$, con valorizzazione delle evidenze romane monetali ed epigrafiche; quindi, e a conclusione di questa lunga fase dedicata a Roma antica, nel 1574 è la volta del Caesar Augustus ${ }^{14}$, seconda opera della serie «imperiale», fondata sempre su documentazione letteraria e numismatica.

Può essere opportuno considerare in breve alcuni aspetti dell'opera che costituisce il precedente immediato al decreto romano di civitas, i Fasti o meglio Fastos magistratuum et triumphorum Romanorum ab urbe condita ad Augusti obitum ex antiquis tam numismatum quam marmorum monumentis restitutos; segue, importante: S.P.Q.R. Hubertus Goltzius Herbipolita Venlonianus dedicavit. Dunque, l'opera è dedicata da Hubertus Goltzius al SENATUS POPOLUSQUe ROMANuS ossia al Senato municipale di Roma, sede simbolica del potere laico antico e attuale, in consapevole e ideale continuità delle politiche del presente storico dello Stato Pontificio con la Roma delle origini. Caratteri e finalità della dedica sono chiariti in una lunga Praefatio (ad Senatum Populumque Romanum), su cui si tornerà. Ma nelle edizioni postume tale atto di ossequio al Senato scompare, presumibilmente perché avvertito come in stretta connessione con le vicende dell'A. e con il suo momento storico, e dunque nei tempi successivi privato dell'attualità e non più degno di memoria. L'edizione dall'Opera omnia goltziana del 1618, riprodotta ancora nel 1644, riporta tale intitolazione: Fasti magistratuum et triumphorum Romanorum ab urbe condita ad Augusti obitum ex antiquis tam numismatum quam marmorum monumentis, segue: Thesaurus item rei antiquariae huberrimus, Huberti

11 A. Bedocchi, Cultura antiquaria e memoria nei volumi della Biblioteca Universitaria di Genova, secoli XVI-XVIII, Genova, 2000, p. 24-5.

12 C. Iulius Caesar siue Historiae imperatorum Caesarumque Romanorum ex antiquis numismatibus restitutae liber primis. Accessit C. Iulii Caesaris vita et res gestae, Huberto Goltzio Herbipolita Venloniano auctore et sculptore, Brugis Flandorum, 1571 (apud Hubertum Goltzium, mense Septembri 1563).

13 Fastos magistratuum et triumphorum Romanorum ab vrbe condita ad Augusti obitum ex antiquis tam numismatum quam marmorum monumentis restitutos S.P.Q.R. Hubertus Goltzius Herbipolita Venlonianus dedicauit, Brugis Flandrorum, 1566 (Brugis Flandorum: excudebat Hubertus Goltzius, 1566, mense Martio).

14 Caesar Augustus siue Historiae imperatorum Caesarumque Romanorum ex antiquis numismatibus restitutae liber secundus. Accessit Caesaris Augusti vita et res gestae. Huberto Goltzio Herbipolita Venloniano ciue Romano auctore et sculptore, Brugis Flandrorum, 1574 (Brugis Flandrorum: excudebat Hubertus Goltzius, 1574, mense Ianuario). 
Goltzii Herbipolitae Venloniani ${ }^{15}$. Anche all'interno manca ogni traccia dell'originaria dedica goltziana al Senato di Roma.

L'edizione del 1566 è molto curata dal suo Autore. Pregnante il valore simbolico del frontespizio, predisposto da Goltzius stesso. Su una sorta di enorme altare, l'iconografia si distribuisce su tre registri: in alto sono tre figure femminili centrali e a lato una divinità maschile con tridente e la lupa romana; al centro due trofei di armi, le spoglie dei nemici vinti in guerra; in basso una serie di personaggi desolati e dallo sguardo basso, tra cui alcuni con le mani legate, evidentemente prigionieri. La decodificazione della raffigurazione è esposta dallo stesso Goltzius nella Frontispicii explicatio, in esametri che rievocano moduli epici e in particolare virgiliani (ad es.: arma, virum, gladios, clypeos, galeasque superbas / [...J). Sotto l'aspetto letterario di un immaginario dialogo tra l'Autore e un Lettore, anzi uno Spectator Rogans per il quale il Goltzius Respondens translittera la simbologia iconografica ed emblematica della raffigurazione che presenta l'opera, leggiamo che la figura femminile centrale riprodotta come divinità armata, tra la Virtù e la Fortuna che si stringono la mano e tra il Tevere e la Lupa che allatta i gemelli è [...] maxima Roma, urbs antiqua, urbs clara, urbs felicissima che con la forza del diritto e delle armi ha dominato il mondo. In basso, è una turba oculos demissa dolentes, con i personaggi legati, che rappresentano [...] populos [...] Veientes, atque Sabinos, / Albanos, Latios, Etruscos, atque Camertes: / inde Fidenates, Volscos, Samnitas, \& Aequos, / Auroncosque, Salentinosque, acresque Faliscos, / Vulsinios, Privernatesque, Tarentinosque $/[\ldots]$, le genti d'Italia assoggettate nella età più antica della espansione romana, tra monarchia e repubblica. Il riferimento alla fase pre-imperiale della storia di Roma, annunciato nel titolo, è ribadito nella sua centralità. Da una scorsa all'Indice (Series eorum quae hoc libro continentur) e al Catalogo dei documenti monetali ed epigrafici, si conferma l'impostazione: protagonista è l'intera storia di Roma, a partire da Romolo e dagli episodi connessi all'età più antica come il ratto delle Sabine, di cui puntualmente si rintracciano reperti numismatici illustrativi, peraltro improbabili per l'epoca ${ }^{16}$. La connessione con l'impero è garantita inoltre dal successivo Catalogo dei consoli dalla morte di Augusto a Giustiniano.

15 Il volume include evidentemente anche il Thesaurus, originariamente del 1579; in esso inoltre si integrano, in aggiunta, i Fastiq. Siculi Denuo Restituti a P. And. Schotto S.I., Antverpiae, 1618 e 1644.

16 Sulla monetazione romana antica, in particolare su aes rude, aes signatum, aes grave, vd. F. PANVINI Rosati, "Monetazione preromana in Italia. Gli inizi della monetazione romana in Italia e la monetazione romano-campana”, in F. PAnvini Rosati, H.A. CAhn (a cura di), La moneta greca e romana, Roma, 2000, p. 79-93. Se non si tratta di falsi, Goltzius può aver riprodotto esemplari numismatici tardi, forse medaglioni commemorativi o "contorniati» tardo-antichi (su cui F. GNeCCHI, Monete romane, Milano, 1935, p. 327-329; A. Alföldi, Die Kontorniat-Medaillons, 3 voll., Berlino, $1976^{2}$ e quindi A. AlföLDI, E. AlföldI, Die Kontorniat-Medaillons, Teil 2, Text, Berlin, 1990). 
Infine, nella Praefatio l'A. dichiara al Senato la propria volontà di ricostruire, al di là e oltre le pubblicazioni della serie «imperiale», la serie dei Fasti romani da riordinare e redigere a condita vestra illustrissima Roma, attraverso la Monarchia e la Repubblica. In rapporto con la storia, rapidamente ripercorsa, della civiltà greca, quella romana mostra maggiore solidità e autorevolezza nelle istituzioni e nel potere, e liberalitas innanzitutto nei confronti della civiltà greca risalta esemplare; ma più in generale, il dominio romano è fondato nô tam vi \& armis, q. benignitate, liberalitate, fide, clemêtia, moderazione, aliis. cognatis virtutibus [... ]; e dai trionfi si può riconoscere la grandezza e la potenza romana. Anche i barbari Odoacre, Teodorico, Alarico, Totila e gli altri hanno sempre mostrato rispetto ed emulazione nei confronti del nomen Romanum, curando l'elogio di Roma in forme ufficiali. Il lungo testo di dedica si conclude all'insegna della continuità: il Senato attuale raccoglie l'eredità dell'antico, con le parole dell'A., eandem Rempublicâ vestro consilio, vestra prudentia administratis. Goltzius ringrazia infine per aver consentito a lui advenam \& peregrinum non solum in Capitolium vestrum admittere, sed etiam intra Palatia vestra et tecta privata, atque adeo in intimos medium vestrarum recessus recepto, iuvandi promovendique instituti mei ergo[...]. Non si allude qui a un ingresso nel Campidoglio in forma ufficiale, ma alla disponibilità e alla humanitas (encomiabile soprattutto nei confronti di uno straniero, quae vestra est ... potissimum exteros...) con cui i Senatori hanno concesso allo studioso fiammingo a Roma (e del soggiorno romano resta documento nella lunga lista dal C.J. Caesar ${ }^{17}$ ) di visionare e apprezzare opulentissimos thesauros numismatici e di antichità anche dalle raccolte personali custodite nelle dimore private. Questo testo (con i contenuti stessi del volume dei Fasti) rappresenta dunque una imprescindibile premessa al senatoconsulto di civitas dell'anno successivo.

I modelli antichi richiamati instaurano relazioni, legami, forme di continuità tra Repubblica e Impero; la connessione con l'operato del Senato municipale si stempera nella storia imperiale. E, come si osserverà più avanti, le politiche papali non risultano incompatibili con i modelli repubblicani evocati; accomunate dal culto per l'antichità e dal collezionismo, esse diventano accondiscendenti, ove non favorevoli, nei confronti di elementi culturali potenzialmente antagonisti e tuttavia riducibili, con tacito accordo della controparte, a spessore puramente erudito rispondente alla logica della condivisione urbana del potere. Gli stranieri, i non-romani, cooptati con la civitas nel corpo civico e nel ceto nobiliare, chiamati a condividere privilegi e doveri con i cittadini di pieno diritto, sono resi grati e deferenti nei confronti dell'autorità romana, che è innanzitutto pontificia, mentre idealmente si identificano nel modulo mitico e insieme attuale di Roma «madre di tutti i popoli».

17 A conclusione dell'opera, il Goltzius pubblica un lungo elenco di personaggi che intende così ringraziare per la disponibilità con cui essi gli avevano consentito la visione e lo studio delle proprie collezioni monetali, con l'indicazione delle località loro sedi, in cui Goltzius aveva sostato nei suoi due viaggi in Europa (1558-60). 
L'ammissione di Hubertus Goltzius alla Romana civitas nel 1567 può dunque porsi agevolmente in rapporto con tale sua produzione, nota all'Europa e all'Italia dei circoli di cultura e di potere, al culmine di una lunga fase di studio e di ricerca sull'antica Roma che egli intendeva fondata su criteri innovativi. La qualifica di civis Romanus si osserva per la prima volta nella firma dell'A. al Caesar Augustus, del 1574: a Huberto Goltzio Herbipolita Venloniano auctore et sculptore ${ }^{18}$, si aggiunge qui ciue Romano. Sfogliando l'opera, dedicata all'imperatore Massimiliano II d'Austria figlio di Ferdinando d'Asburgo ${ }^{19}$, tale qualifica si ripropone in apertura di ogni sezione al ricorrere della firma dell' Auctor, dall'epistola di dedica imperiale e dalla Ad lectorem praefatio, fino alla Vita et Res Gestae Caesaris Augusti e alle dediche encomiastiche degli amici (ad esempio nei versi celebrativi, con dichiarata finalità esegetica del frontespizio dell'opera, di Ludovicus Carrio, di Johannes Posthius Gemerschemius, di Johachimus Tydichius) fino alla epistola laudatoria a firma di Joannes Sambucus Pannonius Tirnaviensis, il medico ungherese umanista, assai noto come autore di Emblemata. Tale qualifica di civitas non viene però altrimenti illuminata o documentata in questa sede.

E tuttavia possibile leggere integralmente il testo del decreto di cittadinanza romana per Hubertus Goltzius emesso dal Senato di Roma perché trascritto e pubblicato dallo stesso beneficiario nella successiva sua opera, Sicilia et Magna Graecia, del 1576, peraltro la prima palesemente dedicata al mondo greco, e poi ancora nel Thesaurus huberrimus, del 1579. Possiamo dunque procedere con l'esame del testo del senatusconsultum quo Hubertus Goltzius civitate Romana donatus est, datato 9 maggio 1567.

\title{
Il decreto di Hubertus Goltzius
}

\author{
EXEMPLAR SENATUSCONSULTI
}

QUO HUBERTUS GOLTZIUS CIVITATE

ROMANA DONATUS EST.

18 Herbipolita per l'origine da Würzburg, latinamente Herbipoli, in Baviera, del padre Roger; Venlonianus perché nato a Venloo, nell'attuale Olanda.

19 Massimiliano d'Asburgo, primogenito dell'imperatore del Sacro Romano Impero Ferdinando I d'Asburgo (cui succede al trono nel 1563, garantendo la continuità del cattolicesimo in Germania, nonostante le personali inclinazioni luterane) rappresentava un referente importante anche per intellettuali e collezionisti. Per lui e per l'imperatore suo padre, l'umanista Jacopo Strada aveva approntato diversi volumi di riproduzioni monetali; a lui lo stesso Strada si rivolge per iscritto con l'obiettivo di difendersi dagli attacchi del suo rivale viennese, lo storiografo imperiale Wolfgang Lazius (D.J. JANSEN, “Antonio Agustín and Jacopo Strada”, in M.H. Crawford [ed. by], Antonio Agustin, between Renaissance and Counter-Reform, London, 1993, p. 211-245, part. 213-4; per un'altra richiesta dello Strada a Massimiliano II, ibid., p. 236-238). 
Stephanus Paparonius, Hieronymus Grossus, Hieron. Bubalus almae Urb. COSS. AD POSTERITATIS MONUMENTUM HIS LITTERIS SCIRE OMNES VOLUMUS INTER PRAECLARA MAIORUM ROMANORUM INSTITUTA ILLUD FUISSE PRAECLARISSIMUM, INSIGNES SCILICET VIRTUTE ET PROBITATE VIROS AUGENDAE REIPUBl. GRATIA, CIVITATE DONARE, UT PRAESTANTISS. CIVIBUS AUCTA, DOMI FORISQUE OMNIUM NATIONUM RES GESTAS SUPERARET. Quorum nos imitatione atQue eXemplo Pyrrho Tario i.v.D. ${ }^{20}$ Hectore Muttinio, VINCENTIO RUBEO, TRIBUS EX QUATTUORVIRIS CIVITATIS DONANDAE FREQUENTIQUE SENATU assistentibus, Hubertum Goltzium Herbipolitam Venlonianum, bene de Romana REPUBL. MERITUM, CUIUSQUE VIRTUS SINGULARISQUE ERGA ROMANUM NOMEN OBSERVANTIA CUM PRAECLARISSIMORUM CIVIUM TESTIMONIO, TUM EDITIS NUPER SCRIPTIS SENATUI PLANE CLARUIT, CIVEM ROMANUM EX S.C. CREAVIMUS, CREATUM IN SENATUM VENIRE, MAGISTRATUS GERERE, IUS FERENDI SUFFRAGII HABERE, SACERDOTIA OBTINERE, BONA EIUS LIBERA ATQUE IMMUNIA ESSE, CETERISQUE ROMANORUM CIVIUM HONORIBUS ATQUE PRIVILEGIIS HONESTARI QUIBUS ILLI FRUUNTUR QUI CIVES ROMANI NATI AUT IURE OPTIMO FACTI SUNT, DECREVIMUS et Censuimus. Ea tamen lege, ut URbem Romam, sicut optimum QUeMQUe CiVEM DECET, SUA VIRTUTE ATTOLLAT, FIDELITER A QUOCUMQUE VEXANTE TUEATUR, IURA PONTIFICIA ROMANIS CONCESSA, ATQUE SENATUS CONSULTA OBSERVET, ROMANOSQUE OMNI DILECTIONE PROSEQUATUR. DANTES SCRIBIS SENATUS P.Q.R. AUCTORITATEM, FIDEI ERGO ATQUE AD PERPETUAM POSTERITATIS MEMORIAM, ACTA HAEC ATQUE DECRETA IN PUBLICAS TABULAS SUBSCRIPTIONE REFERRE CUM SIGILLI S.P.Q.R. APPENDICE. DATUM IN PALATIO CAPITOLINO VII IDUS MAI M.D.LXVII.

IULIUS HOROLOGIUS SCRIBA S.P.Q.R.

HORATIUS FUSCUS SACRI S.P.Q.R. SCRIBA.

"Copia del senatoconsulto

con cui a Hubertus Goltzius fu conferita la cittadinanza romana

Noi, Consoli della benevola ${ }^{21}$ Città, Stefano Paparonio, Geronimo Grosso, Geronimo Bubalo, a monumento della posterità, con questo testo vogliamo che tutti conoscano tra le più illustri istituzioni degli avi (dei) Romani quella che fu la più illustre in assoluto, di conferire la cittadinanza a uomini chiaramente stimati per valore e probità per rendere più grande lo stato, affinché onorato da questi uomini nobilissimi in patria e fuori superi le imprese di tutte le genti.. ${ }^{22}$ Tra essi noi per l'emulazione

20 Iuris utriusque doctor, di diritto civile e canonico.

21 Alma è propriamente da intendersi come «nutrice», "datrice di vita», in tal senso "benevola». L'espressione Alma Urbs (come Alma Roma) è convenzionale e di largo uso, impiegata anche sulla medaglia incisa in occasione del giubileo del 1500: così A. GIARDINA, A. Vauchez, Il mito di Roma. Da Carlo Magno a Mussolini, Roma-Bari, 2008, p. 91-2.

22 Con il simbolo - ho inteso evidenziare le tre sezioni costitutive, a mio avviso, del testo. 
e per l'esempio ${ }^{23}$, essendo presenti Pirro Tario, dottore in diritto canonico e civile, Ettore Muttinio, Vincenzo Rubeo, tre dei quattuorviri preposti al conferimento della cittadinanza e il senato numeroso, abbiamo nominato cittadino romano per decreto del senato Hubertus Goltzius Herbipolita Venloniano, benemerito dello stato romano, il valore del quale e la singolare devozione nei confronti del nomen romano sia per la testimonianza dei cittadini più illustri sia per gli scritti da poco editi, al senato fu ben chiaro, (egli cittadino romano) una volta nominato abbiamo stabilito e decretato che venga in senato, ricopra le magistrature, abbia il diritto di voto, ottenga cariche ecclesiastiche, siano i suoi beni liberi ed esenti da tributi ed egli sia onorato con gli altri onori e privilegi propri dei cittadini romani dei quali fruiscono coloro che nacquero cittadini romani o lo divennero di pieno diritto. Tuttavia per quella legge (si prescrive) che, come si addice a tutti i migliori cittadini, egli elevi la città di Roma con la sua virtù, fedelmente la tuteli da chiunque l'attacchi, osservi le leggi pontificie concesse ai Romani e i decreti del senato, tratti i Romani con tutto l'apprezzamento possibile.m Dando noi l'autorità agli scribi del Senato e del Popolo Romano, a garanzia e dunque a perpetua memoria della posterità, questi atti e decreti si riportino firmati nei registri ufficiali con l'apposizione del sigillo S.P.Q.R. conferito sul palazzo del Campidoglio il VII giorno delle idi di maggio 1567.

Giulio Orologio, scriba del S.P.Q.R.

Orazio Fusco, scriba del santo/sacro S.P.Q.R.».

Si evidenziano, comuni alla intera categoria dei Privilegia Civitatis o Civilitatis $^{24}$, chiari richiami al complesso istituzionale dell'antica Roma pre-cristiana e pre-papale: oltre al costante riferimento alla romana respublica, oltre al richiamo ai consules $^{25}$, al

23 L'espressione imitatione atque exemplo parrebbe indicare il motivo dell'attribuzione del privilegio: nell'accezione più ovvia si renderebbe "per l'emulazione e per l'esempio», sottintendendo un'espressione del tipo «dei nostri avi» o simile, di cui peraltro è documentazione nel decreto di cittadinanza del Montaigne, qui esaminato di seguito: [...] Nos, maiorum nostrorum exemplo atque auctoritate permoti [...]. Un'altra resa potrebbe essere "per l'imitazione e per la rappresentazione» allusiva agli studi di antichità del Goltzius e più specificatamente alle speciali sue competenze nel riprodurre (imitatio) e nel raffigurare, disegnare, copiare (exemplum) gli elementi della cultura materiale antica, soprattutto numismatici.

24 Privilegium Romanae Civilitatis è la nomenclatura costante nelle minute dei decreti. Nell'impiego la civitas (- civis) tende a sovrapporsi e a coincidere con la civilitas (- civilis): vd. A. Ernout, A. Meillet, Dictionnaire étymologique de la langue latine. Histoire des mots, Paris, $1985^{4}$, s.v. cīvis, p. 124 . Il campo semantico di civilitas è propriamente più ampio e dunque meno tecnico, nell'accezione di una "cittadinanza» da intendersi come d'uso più informale.

25 La storia di Roma repubblicana è connotata dalla costante dialettica, talora contrastiva, tra l'autorità dei consules e quella del senatus (su cui ad es. F. De MarTino, "Il Senato Romano. Introduzione", in Il Senato nella storia. I. Il Senato nell'età romana, Roma, 1998, p. 6-10, 27); successivamente prevarrà la tensione tra princeps/dominus e senatus. Nel nostro caso i consoli sono i promotori della proposta di assegnazione, ma essa non potrebbe aver sèguito 
senatus e ai senatusconsulta ${ }^{26}$, è la stessa civitas onoraria ${ }^{27}$ a essere presentata come l'istituzione più illustre della Roma degli avi. Vogliono rievocare in forma più generica magistrature e realtà romane antiche i Quattuorviri Civitatis Donandae ${ }^{28}$, gli stessi Scribae Senatus e anche il sigillo S.P.Q.R. (specificato in altri decreti come d'argento, più raramente d'oro ${ }^{29}$ ) a chiusura, sul quale veniva raffigurata Roma triumphans spesso super

senza la ratifica del senato; dunque i due istituti si integrano nell'operato, ma i consoli ricoprono un ruolo più attivo e propositivo ove non decisionale.

26 L'istituzione senatoria sopravvive alla fine dell'Impero Romano d'Occidente, ma la sua decadenza continua irrimediabile: ancora a inizio VII sec. d.C. il senato, costituito da un esiguo numero di laici, si riuniva associandosi alle manifestazioni ecclesiastiche presiedute dal Papa, cui era vincolato anche nella propaganda. Successivamente quasi scomparso nei testi, riaffiora poi nella nomenclatura di assemblee romane di diverso carattere in nuove realtà storico-politiche. Il richiamo alla continuità con il passato antico di Roma aeterna assumerà puro valore ideologico e strumentale, con finalità di auto-proclamazione da parte dei nuovi ceti dominanti. Sulla questione vd. tra gli altri L. Cracco RugGini, "Il Senato tra due crisi (III-VI secolo)", in Il Senato nella storia I, p. 223-375, part. 373-375; sul senatusconsultum come ratifica formale della posizione assunta dal senato sulle differenti questioni, sulla procedura di promulgazione, sul (discusso) valore normativo vd. F.M. D'Ippolito, "Le origini del Senato e la prima età repubblicana", in Il Senato nella storia I, p. 29-83, part. 73-78, con precedente bibliografia.

27 Per la quale un parallelo possibile è con la civitas per honorem o per meriti individuali nella Roma antica; per i magistrati locali allo scadere del mandato c'era la possibilità di divenire cittadini romani, concessione estensibile all'intero nucleo familiare, dai genitori e figli ai discendenti; questa civitas onoraria, assegnata spesso per intervento dell'imperatore stesso, era celebrata con orgoglio in dediche e iscrizioni (A.N. SHERWIN-WHITE, The Roman Citizenship, Oxford, 1996 [1939]; P. Donati Giacomini, G. Poma, Cittadini e non cittadini nel mondo romano. Guida ai testi e ai documenti, Bologna, 1996, p. 149 s.). Tale cittadinanza aveva carattere individuale e strettamente personale, e poteva, come la civitas in genere, designare anche collettività, come tribù, comunità o gruppi sociali (G. CRIFò, Civis. La cittadinanza tra antico e moderno, Roma-Bari, 2005, p. 33).

28 La concessione della cittadinanza romana agli alleati latini e italici a partire dalla guerra sociale (91-89 a.C.) si accompagnava a un'organizzazione fondata sullo schema del municipium con una costituzione uniformata che faceva capo quasi ovunque a un collegio di quattro magistrati, appunto Quattuorviri (Quattuorviri Iure Dicundo e Quattuorivi Quinquennales, due e due, con funzioni censorie e anche consolari): U. LAFFI, "I Senati locali nello stato municipale e nel I sec. d.C.", in Il Senato nella storia I, p. 377-398, part. 377-381. Per i Quattuorviri Civitatis Donandae e anche per gli Scribae S.P.Q.R. non parrebbero risultare puntuali corrispondenze. Scribae a Roma erano i notai o gli impiegati stipendiati dallo stato e utilizzati nella compilazione del bilancio pubblico, nella trascrizione delle leggi e nella registrazione degli atti dei funzionari statali. Divisi in compagnie o classi (decuriae), erano attribuiti per sorteggio ai magistrati con mansioni di segretari. Così, dai funzionari cui erano assegnati, prendevano il nome di [Scribae] Quaestorii, Aedilicii, Praetorii.

29 L'indicazione relativa al sigillo si intende ovviamente finalizzata alla forma definitiva e solenne del decreto, in pergamena e con cordone in seta dorata; anche in seguito questo resterà il bollo ufficiale delle concessioni di cittadinanza e anche, insieme, di nobiltà, 
spoliis. Con la cittadinanza, Goltzius acquista honores atque privilegia precisati come quelli propri dei cives Romani nati aut iure optimo facti (categoria quest'ultima che nella Roma antica designava i cittadini romani di pieno diritto, spesso insediati nelle colonie e distinti dai cives sine suffragio ${ }^{30}$ ). Sono i consules, indicati nominalmente (tre, come usuale per questa fase ${ }^{31}$, Stephanus Paparonius, Hieronymus Grossus, Hieronymus Bubalus), a promuovere l'iniziativa, a dichiararne le finalità, a dettarne le modalità; difatti essi si esprimono in prima persona plurale nell'intero testo. Ma la proposta non potrebbe avere realizzazione senza l'approvazione del senato con la promulgazione del senatusconsultum, il decreto senatorio, forma ufficiale del privilegium; dunque, il conferimento vero e proprio della cittadinanza viene demandato al senato. La dialettica esclusiva consoli-senato sembra ispirarsi alla storica tensione del passato repubblicano, pre-papale e anche pre-imperiale, senza spiragli per eventuali elementi propri della cristianità. Viene inoltre precisata l'attività dei Quattuorviri civitatis donandae, presenti in tre, di cui pure si riferiscono i nomi (Pyrrhus Tharius, Hector Muttinius, Vincentius Rubeus), magistratura intermedia e strettamente preposta alla nomina del civis.

Il decreto può dunque ripartirsi sostanzialmente in tre sezioni.

A) La premessa, con l'intervento dei Consoli; il motivo propagandistico della vetustà del privilegio indicato come il più illustre istituto tra quelli degli avi, a ulteriore raccordo con Roma antiqua; l'indicazione delle finalità (ad monumentum posteritatis; [...] [reipublicae] omnium nationum res gestas superaret) e dei caratteri dell' institutum praeclarissimum: assegnando la cittadinanza romana onoraria a personalità di spessore culturale universalmente riconosciuto, stimate virtute et probitate, viene ad incrementarsi il prestigio della stessa Urbs.

quindi del solo patriziato: E. MorI, “Tot reges in urbe Roma quot cives”. Cittadinanza e nobiltà a Roma tra Cinque e Seicento", in Il Comune di Roma. Istituzioni locali e potere centrale nella capitale dello Stato Pontificio, Roma moderna e contemporanea 2 (1996), p. 379-401, part. 391.

30 Ad es. vd. A.N. Sherwin-White, The Roman Citizenship, p. 38-58, 200-214, 304-6, 317, 336; G. Mousourakis, The Historical and Institutional Context of Roman Law, Auckland, 2003, p. 27, 67; C. Williamson, The Laws of the Roman People. Public Law in the Expansion and Decline of the Roman Republic, University of Michigan Press, 2005, p. 197 s.

31 M. Fischer, W. Pedrotti, Le città italiane nel medioevo. La geografia delle Signorie, Roma, 1997, p. 43: già i Consigli dei Comuni medievali eleggevano in molte città magistrati con autorità di governo, denominati Consoli; nonostante il palese richiamo alla magistratura romana nella società comunale i consoli erano generalmente più di due; tre a Roma, potevano raggiungere altrove anche il numero di venti o trenta. Un dettaglio: nella citata lista dal C.J. Caesar di Goltzius compare per Roma anche un tale Hieronymus Paparonus, forse un consanguineo del console Stefano del decreto goltziano. 
B) Il decreto proprio, con la designazione del civis, solo ora indicato nominalmente, da parte dei Consoli e per il tramite dei IVviri civitatis donandae, e quindi la motivazione del privilegio, unitamente a un elenco di diritti e di obblighi così fatti propri. Tale motivazione viene espressamente connessa con le opere dello stesso Goltzius in cui egli ha mostrato rispetto e cura per il nomen Romanum e con le attestazioni in suo favore di cives praeclarissimi, nei quali potrebbe forse riconoscersi la commissione di funzionari eletti ad acquisire informazioni sulle qualità dei futuri cittadini romani già attiva nel 1519 e rinnovata periodicamente, dal 1580 ufficialmente registrata negli Statuti ${ }^{32}$. In grazia di tale civitas a Hubertus Goltzius si assegnano ampi privilegi, attivi e passivi, di cui segue elenco: integrazione nel senato municipale, possibilità di ricoprire magistrature e cariche sacerdotali, diritto di voto, esenzione dai tributi sui beni, onori e privilegi ulteriori non precisati. Alla classificazione dei diritti fanno seguito i doveri più genericamente enunciati, nella sostanza riconducibili all'apprezzamento ufficiale nei confronti dell'Urbs, con proponimento di tutela a quocumque vexante e con obbligo di rispetto, nell'ordine, degli iura pontificia Romanis concessa e dei senatusconsulta. Attraverso il richiamo all'osservanza delle sue leggi, la sovranità del papa, massima autorità politica dello Stato Pontificio ma anche suprema autorità religiosa per la cristianità, entra così manifestamente nel formulario repubblicano e laico del decreto evocante la Roma precattolica e pre-pontificia, in una sorta di attualizzazione conclusiva che ristabilisce nella «corretta» direzione l'equilibrio tra «il Comune antico» e la supremazia papale.

C) La clausola di chiusura, con cui i consules accordano agli scribae l'auctoritas di trascrivere e sottoscrivere gli acta haec atque decreta in publicas tabulas, con luogo (il palatium del Campidoglio), data e firma dei due scribasenato, Julius Horologius e Horatius Fuscus.

\section{Il decreto di Michel de Montaigne. Un raffronto possibile}

Per il XVI secolo un utile confronto con il nostro testo è rappresentato dal noto decreto di cittadinanza romana per un illustre esponente della cultura d'oltralpe, Michel de Montaigne. Il privilegio data 13 marzo 1581, quattordici anni dopo il senatusconsultum di Goltzius. Lo scribasenato di Goltzius, Horatius Fuscus ${ }^{33}$, è firmatario pure per Montaigne e anche in questo caso il testo è a noi noto per la trascrizione e la pubblicazione da parte dell'assegnatario. È difatti lo stesso Montaigne a riprodurre il suo decreto nel terzo libro degli Essais, dichiarando di aver così inteso soddisfare una eventuale

32 Mori, Tot reges, p. 390. Tale commissione durava in carica da uno a quattro anni.

33 Il Fusco, in qualità di notaio capitolino, pare avesse ottenuto il Segretariato delle Fonti come incarico vitalizio a partire dal 1581 e dopo una disputa con Ermete Gracco (D. SINISI, "I Notari Magistrorum Stratarum nel'500", Roma moderna e contemporanea IV, 2 [1996], p. 363-378, part. 373). 
curiosità del lettore e descrivendo la bolla nel suo lussuoso aspetto esteriore ("pomposa in sigilli e lettere dorate») ma nel contempo ammettendo la propria umana vanità ${ }^{34}$ :

Essais III 9

"Parmy ses faveurs vaines, je n'en ay point qui plaise tant à ceste niaise humeur, qui s'en paist chez moy, qu'une bulle authentique de bourgeoisie romaine: qui me fut octroyée dernierement que j'y estois, pompeuse en seaux, et lettres dorées: et octroyée avec toute gratieuse liberalité. Et par ce qu'elles se donnent en divers stile, plus ou moins favorable: et qu'avant que j'en eusse veu, j'eusse esté bien aise, qu'on m'en eust montré un formulaire: je veux, pour satisfaire à quelqu'un, s'il s'en trouve malade de pareille curiosité à la mienne, la transcrire icy en sa forme [...]».

«Tra i suoi favori vani, io non ne ho alcun altro che piaccia tanto a questo stupido umore che io provo, che una bolla autentica di borghesia (= cittadinanza) romana: che mi fu concessa nell'ultimo periodo che io vi sono stato, enfatica in sigilli e lettere dorate: e concessa con graziosissima liberalità. E dal momento che esse si danno in diverso stile, più o meno encomiastico: e che prima che ne avessi viste, io sarei stato ben contento, che mi si fosse mostrato un formulario: voglio, per soddisfare qualcuno, se se ne trova (uno) malato di curiosità simile alla mia, trascriverla qui nella sua forma.[...]».

Quod Horatius Maximus, Martius Cecius, Alexander Mutus, almae urbis Conservatores, de Illustrissimo viro Michaele Montano, equite Sancti Michaelis, et a cubiculo Regis Christianissimi, Romana Civitate donando, ad Senatum retuleRUNT, S. P. Q. R. DE EA RE ITA FIERI CENSUIT.

CUM VETERI MORE ET INSTITUTO CUPIDE ILLI SEMPER STUDIOSEQUE SUSCEPTI SINT, QUI, VIRTUTE AC NOBILITATE PRAESTANTES, MAGNO REIP. NOSTRAE USUI ATQUE ORNAMENTO FUISSENT VEL ESSE ALIQUANDO POSSENT, Nos, MAIORUM NOSTRORUM EXEMPLO ATQUE AUCTORITATE PERMOTI, PRAECLARAM HANC CONSUETUDINEM NOBIS IMITANDAM AC SERVANdam fore Censemus. Quamobrem, QuUm Illustrissimus Michael Montanus, Eques sancti Michaelis, et a Cubiculo Regis Christianissimi, Romani nominis studiosisSIMUS, ET FAMILIAE LAUDE ATQUE SPLENDORE ET PROPRIIS VIRTUTUM MERITIS DIGNISSIMUS Sit, Qui summo Senatus Populique Romani Iudicio aC STudio in Romanam Civitatem adsciscatur, Placere Senatui P.Q.r. Illustrissimum Michaelem Montanum, rebus OMNIBUS ORNATISSIMUM ATQUE HUIC INCLYTO POPULO CHARISSIMUM, IPSUM POSTEROSQUE IN Romanam CiVITATEM ADSCRIBI ORNARIQUE OMNIBUS ET PRAEMIIS ET HONORIBUS QUIBUS illi fruUntur qui Cives Patricitque Romani nati aUt JURe optimo facti SUNT. IN QUO CENSERE SENATUm P.Q.R. SE NON TAM Illi IUS Civitatis largiri QUam Debitum TRIBUERE, NEQUE MAGIS BENEFICIUM DARE QUAM AB IPSO ACCIPERE QUI, HOC CIVITATIS MUNERE ACCIPIENDO, SINGULARI CIVITATEM IPSAM ORNAMENTO ATQUE HONORE AFFECERIT. QuAm QUidem S. C. AUCTORITATEM IIDEM CONSERVATORES PER SENATUS P.Q.R. SCRIBAS IN ACTA REFERRI, ATQUE IN CAPITOLII CURIA SERVARI, PRIVILEGIUMQUE HUJUSMODI FIERI, SOLI-

34 Alla vanità umana è dedicato l'intero terzo libro. 
TOQUE URBIS SIGILLO COMMUNIRI CURARUNT. ANNO AB URBE CONDITA CXC.CCC.XXXI. POST ChrisTUM NATUM M.D.LXXXI. III. IDUS MARTII.

HORATIUS FusCus, SACRI S. P. Q. R. SCRIBA.

Vincen. Martholus, SACri S. P. Q. R. SCRIBA.

«In base a quanto riferirono al Senato, Orazio Massimo, Marzio Cecio, Alessandro Muto, Conservatori della benevola Città, sulla concessione della cittadinanza romana all'illustrissimo Michel de Montaigne, cavaliere dell'Ordine di San Michele e gentiluomo della camera del Re Cristianissimo, il Senato e il Popolo Romano hanno così decretato che si faccia relativamente a tale argomento: poiché per antica usanza e istituzione sono sempre stati accolti fra noi con calore e zelo coloro che, eminenti per virtù e nobiltà, fossero stati di grande servigio e onore per il nostro stato o potevano esserlo un giorno, Noi, mossi dall'esempio e dall'autorità dei nostri antenati, crediamo nostro dovere imitare e conservare tale nobile consuetudine. Quindi, poiché l'illustrissimo Michel de Montaigne, cavaliere di San Michele e gentiluomo della camera del Re Cristianissimo, amantissimo del nome romano, sia per la nobiltà e il lustro della sua famiglia sia per le eminenti virtù personali è oltremodo degno di essere ammesso alla cittadinanza romana per supremo giudizio e volontà del Senato e del Popolo Romano, è piaciuto al Senato e al Popolo Romano che l'illustrissimo Michel de Montaigne, adorno di ogni merito e assai caro a questo nobile popolo, fosse iscritto alla cittadinanza romana, lui e la sua discendenza, e ammesso e adornato di tutti i privilegi e gli onori di cui godono coloro che nacquero cittadini e patrizi di Roma o lo divennero a giustissimo diritto. Nel che il Senato e il Popolo Romano ritennero non tanto di accordargli un diritto di cittadinanza quanto di pagare un debito, non tanto di dare un beneficio quanto di riceverlo da lui stesso che, accettando questo diritto di cittadinanza ha dato singolare lustro ed onore alla città stessa. I I medesimi Conservatori invero hanno fatto riportare agli atti questo senatoconsulto dagli scribi del Senato e del Popolo Romano, conservarlo nella curia del Campidoglio, e hanno fatto redigere questo atto e apporvi secondo la regola il sigillo della città. L'anno 2331 dalla fondazione di Roma; il 1581 dalla nascita di Cristo, 13 di marzo.

Orazio Fusco, Scriba del santo/sacro Senato e del Popolo Romano

Vincenzo Martolo, Scriba del santo/sacro Senato e del Popolo Romano»

"N'estant bourgeois d'aucune ville, je suis bien aise de l'estre de la plus noble qui fut et qui sera onques. Si les autres se regardoient attentivement, comme je fay, ils se trouveroient comme je fay, pleins d'inanité et de fadaise: De m'en deffaire, je ne puis, sans me deffaire moy-mesmes. Nous en sommes tous confits, tant les uns que les autres. Mais ceux qui le sentent, en ont un peu meilleur compte: encore ne sçay-je.[...]”.

"Non essendo cittadino di alcuna città, sono ben lieto di esserlo della più nobile che fu e che sarà mai. Se gli altri si analizzassero con attenzione, come io faccio, si scoprirebbero come succede a me, pieni di vanità e stoltezza: non posso liberarmene senza distruggere me stesso. Ne siamo tutti impregnati, tanto gli uni che gli altri. Ma 
coloro che se ne accorgono ne sono un po' più esenti: per quanto io non ne sia certo. $[\ldots] »$.

In una più ampia e critica riflessione sulla vanità umana, il decreto di civitas di Montaigne fa riferimento a magistrature municipali sostanzialmente analoghe a quelle richiamate nel precedente decreto, ma con scarse similarità nella titolatura e nel lessico e assonanze saltuarie negli impieghi delle locuzioni tratte dall'abituale formularità amministrativa. Anche in questo caso il testo è introdotto dal richiamo nominale ai tre Consoli, indicati nominalmente e detti Conservatores, ugualmente almae urbis. Essi promuovono di fronte al Senato la Romana Civitas, approvata con senatoconsulto e provvedono a che la trascrizione del decreto senatorio, redatto e custodito sul Campidoglio, venga approntata dagli scribasenato. Diversamente dall'altro privilegio, qui è il Senato (nella sigla S.P.Q.R.) ad accogliere la relazione dei Conservatori-Consoli sulla concessione della civitas onoraria e, parrebbe, a «dettare» il testo, ma sono sempre i Conservatores a prescrivere la trascrizione dell'atto e la sua archiviazione nella Curia Capitolina. Dunque, nella sostanza la dinamica resta similare: proposta dei Consoli, approvazione e decreto del Senato, trascrizione del privilegio.

Anche questo testo si mostra costituito da tre sezioni.

A) La premessa, con i nominativi dei Conservatori, in cui si richiamano i caratteri e le finalità dell'istituzione e si introduce il nuovo civis, subito presentato con i suoi titoli: Cavaliere dell'ordine di San Michele ${ }^{35}$, il cui Gran Maestro era il re di Francia, e a cubicolo Regis Christianissimi, ossia Gentiluomo Ordinario della Camera del Re più religioso nel cristianesimo, ancora il re di Francia. Del resto Michel de Montaigne opera alla corte di Carlo IX di Valois (re di Francia fino al 1574), che risulta averlo insignito del titolo di Gentiluomo di Camera nel 1571 ${ }^{36}$; collabora successivamente con Enrico III (fratello dello stesso Carlo IX e re fino al 1589) ${ }^{37}$ e quindi con Enrico IV (re di

35 Ordine cavalleresco fondato nel 1469 dal re Luigi XI in competizione con l'Ordine del Toson d'Oro. I 36 cavalieri prestavano giuramento di fedeltà al re, loro Capo Supremo; l'Arcangelo san Michele era ritenuto il protettore della Francia dal momento che l'aveva tutelata contro l'Inghilterra nella Guerra dei Cent'anni. Il Montaigne era stato insignito del cavalierato nel 1571 dal re Carlo IX. Sull'ordine di Saint-Michel si vd. ora B. DE FaUCONPRET, Les chevaliers de Saint-Michel, 1665-1790, Paris, 2007.

36 G. Tomasi di Lampedusa, Invito alle lettere francesi del Cinquecento, Milano, 1979 (Montaigne: p. 95-112), p. 99; I.G. Burnham, G. Norton (ed. by), A Handbook to the Essays of Michel De Montaigne, Whitefish (Montana), 2005, p. 1762; L.-A. Colliard, Montaigne. Nouvelles lumières sur l'auteur des Essais, Fasano, 2007, p. 31. Il Montaigne da sindaco di Bordeaux divenne consigliere al Parlamento della città, esercitando le proprie funzioni con diverse missioni alla corte di Francia e per molti anni.

37 N. Zemon Davis, Il dono. Vita familiare e relazioni pubbliche nella Francia del Cinquecento, Milano, 2002 [2000], p. 101. 
Francia dal 1589 al 1610, già re di Navarra dal 1577, ugonotto convertitosi una volta salito al trono) che nel 1577, ancora re di Navarra, lo nomina anch'egli gentilhomme de sa Chambre ${ }^{38}$. Dal momento che il nostro privilegio data $1581 \mathrm{e}$ che il re in questione è christianissimo, il riferimento si può intendere plausibile per Carlo IX e anche per Enrico III.

A ogni modo, tali qualifiche prospettano il personaggio come straordinario, per nobiltà di natali oltre che per le onorificenze e i legami privilegiati con la monarchia francese: illustrissimus vir, più avanti è tra i virtute ac nobilitate praestantes e ancora, gli si assegnano gli onori dei cives patriciique nati [...] e non solo dei cives, come invece per Hubertus Goltzius. In virtù di tali elementi l'attribuzione al Montaigne della civitas onoraria si propone come finalizzata a rendere più illustre l'Urbs, veteri more et instituto, sull'esempio e l'autorità dei maiores.

B) La sezione centrale, che contiene il decreto vero e proprio, con le motivazioni del privilegium, in parte anticipate nella premessa (nobiltà familiare, virtù personali). In essa si elencano i diritti del civis unitamente alla considerazione, a carattere convenzionale, che con tale approvazione il Senato non ritiene di accordare un diritto quanto di pagare un debito.

C) La clausola di chiusura, consistente nella disposizione dei Conservatori agli scribi di trascrizione del decreto con sigillo e data, indicata secondo la doppia modalità, laica e cristiana ( $a b$ Urbe condita e a Christo nato), il che è riscontrabile per questa fase pure in altri decreti ${ }^{39}$. Anche il testo di Goltzius si concludeva con datazione «mista», ma senza tale espressa distinzione, con giorno-mese secondo il calendario romano, anno secondo l'era cristiana. I due scribae sottoscrivendo si dichiarano entrambi sacri S.P.Q.R., «del santo (o sacro) Senato e del Popolo Romano». Per la formula si potrebbe forse richiamare a confronto la Sacra Romana Respublica di Cola di Rienzo del precedente secolo, scevra da riferimenti religiosi. Nel nostro caso, se sacer (S.P.Q.R.), riferito al Senato, ha valore di «santo», la qualifica rende palese l'egida pontificia sull'istituto municipale e la presenza contestuale del potere del papa, evocata anche dalla datazione; se l'accezione corretta è però "sacro», nel senso di «inviolabile», viene evocata la sacralità laica dell'istituzione, in tal caso nel solco della tradizione classica. Tale ambiguità semantica ha comunque una sua funzionalità. Che già nel decreto per Goltzius, Horatius Fuscus si fosse firmato sacri [S.P.Q.R.], lungi dall'indicare l'appartenenza a un ulteriore senato a predominante carattere religioso (cardinalizio, peraltro esistente ${ }^{40}$ ),

38 N. Zemon Davis, ibid.

39 Registro di Privilegi di Cittadini Romani Creati, Cred. I, tomo 1, ad es. 151v (per Julius Jacobonius, 1580: a nativitate), consultabile presso l'Archivio Storico Capitolino.

40 Il collegio dei cardinali è l'organismo parallelo al Senato laico in ambito ecclesiastico. "Senatori della Chiesa Universale", i cardinali costituiscono una sorta di Senato pontificio ben più autorevole di quello municipale per peso politico ma di integrità discussa, anche 
potrebbe spiegarsi come una sorta di ininfluente opzione da una formula standard a discrezione degli scribi: da una scorsa alle minute dei privilegia, conservate presso l'Archivio Storico Capitolino ${ }^{41}$, su cui si tornerà, si evince che lo stesso Julius Horologius, scriba S.P.Q.R. nel decreto di Goltzius, in altri privilegia civilitatis del $1560^{42}$ si firma scriba sacri S.P.Q.R.; e mentre nel decreto del Montaigne entrambi gli scribasenato si definiscono sacri S.P.Q.R., essi stessi, spesso in coppia, non sempre rispettano tale modalità di sottoscrizione, pur nel loro caso predominante ${ }^{43}$.

Diversamente da Michel de Montaigne, di Hubertus Goltzius non si elogiano origini gentilizie, peraltro non altrimenti documentate, né suoi eventuali autorevoli titoli onorifici: pur nel riprodursi formulare dei modelli, questi privilegi contengono elementi di originalità e attualità connessi alle individuali caratteristiche del destinatario. Goltzius è tra gli insignes virtute et probitate viri, tra i praestantissimi cives, ma non tra gli illustres o nobiles, pur essendo personaggio assai noto e ben introdotto negli ambienti europei di corte e di potere del XVI secolo (come tra l'altro comprova, ancora una volta, il suo stesso elenco dal C. Julius Caesar). L'ideale equiparazione del suo stato sociale acquisito è con i cives Romani e non con i cives patriciique Romani (nati).

Nel raffronto, la sezione centrale dei due decreti si presenta di similare ampiezza complessiva ma risulta più articolata per il Goltzius, con i meriti del civis e l'elenco dei suoi diritti/doveri. Inoltre in questo suo decreto interviene la magistratura intermedia dei Quattuorviri Civitatis Donandae, raramente evocata in altri privilegi ${ }^{44}$ e comun-

perché diviso in fazioni sotto controllo del Pontefice, della nobiltà romana e delle principali potenze europee che tentano di condizionare la scelta di un nuovo papa anche molto prima della sua designazione ufficiale (A. MenNiti Ippolito, «I due "senati" del sovranopontefice: il Collegio dei cardinali e il Municipio romano in età moderna", in Il Senato nella storia, I, p. 453-490; ID., Il governo dei papi nell'età moderna. Carriere, gerarchie, organizzazione curiale, Roma, 2007, p. 50-55).

41 Sui fondi contenuti nell'Archivio Storico Capitolino e sulla sua storia: L. GuAsco, L'Archivio Storico Capitolino, Roma, 1946; E. MorI, "Gli archivi gentilizi”, p. 28-31 e in generale i contributi ne Il Comune Antico e il suo archivio, Comune di Roma, Assessorato alla Cultura, Centro di Coordinamento Didattico, Roma, 1989; P. Pavan, "Tra erudizione e storiografia: il caso dell'Archivio Capitolino”, p. 101-113 e M. FranCESCHINI, "L’Archivio Storico Capitolino e il problema degli strumenti di ricerca", p. 278-293, in Archivi e archivistica a Roma dopo l'unità. Genesi storica, ordinamenti, interrelazioni, Atti del Convegno (Roma, 12-14 marzo 1990), Roma, 1994.

42 Registro di Privilegi di Cittadini Romani Creati, Cred. I, tomo 1, 37, 38, 39, 40, 41, 42, 43: 8 decreti, in cui egli si definisce "scriba sacri senatus Almae Urbis, cum sigillo senatus P.Q.R. appenso»; ma da p. 44, a partire ancora dal 1560, Horologius si firma anche solo scriba senatus P.Q.R, aggiungendo la descrizione delle raffigurazioni ufficiali di Roma sul sigillo: "cum appensione sigilli argentei solidi a cuius altero later' erant arma SPQR ab altero erat Roma triumphans sedens sup $=$ spoliyss.

43 Registro di Privilegi di Cittadini Romani Creati, Cred. I, tomo 1, 154 e passim.

44 Come nel caso del decreto per Petrus e Aloysius Maior, del 1561, Registro di Privilegi di Cittadini Romani Creati, Cred. I, tomo 1, 67 r. 
que mancante per Montaigne. Qualificato come bene de Romana republ. meritum [H. Goltzium], dalla virtus singularisque erga Romanum nomen observantia, per il Senato egli si distingue editis nuper scriptis. Nessun cenno agli scritti del Montaigne nel suo privilegio.

Dettagliati i diritti del civis Goltzius: egli può prendere parte alle sedute del senato, ricoprire magistrature, avere il diritto di voto, conseguire sacerdotia, avere proprietà di beni non soggetti a imposta e infine, complessivamente, godere degli onori e dei privilegi dei cives romani nati (o divenuti tali) di pieno diritto (optimo iure). I suoi obblighi, più generici, consistono nell'esaltare e tutelare l'Urbs dagli attacchi alla Romana virus, nel rispetto di iura pontificia e senatusconsulta e infine nel trattare i Romani stessi con il più grande apprezzamento.

Nella corrispondente sezione del decreto di Montaigne, qualificato come Romani nominis studiosissimus, accanto al valore delle virtutes personali, concetto evocato anche più oltre (rebus omnibus ornatissimum), e al rinnovato richiamo alla nozione di nobiltà familiare, si aggiunge la predilezione del popolo romano nei suoi confronti (atque huic inclyto populo charissimum), elementi tutti motivanti il giudizio senatorio di idoneità. Come si accennava, non si menzionano meriti intellettuali connessi alle opere del Montaigne, come se esse nella Roma pontificia del Cinquecento non fossero conosciute o (piuttosto) non apprezzate. A questo punto si introduce il formulario dei diritti e degli obblighi inerenti la nuova condizione di civis, ma l'esposizione diviene generica, per nulla confrontabile con la parallela sezione del privilegio di Goltzius. Si legge difatti: ornarique omnibus et praemiis et honoribus, quibus illi fruuntur, qui cives patriciique Romani nati [...], o che sono tali iure optimo (in tal caso, come per il Goltzius); a tale approssimazione, si aggiunge la mancanza di ogni riferimento agli obblighi. Anche se nel Voyage ${ }^{45}$ e, si è detto, negli Essais, il Montaigne descrive il diploma ricevuto come celebrativo e di grande effetto, magnifico per sigilli e lettere d'oro, dalla tipologia ricercata e non dissimile dall'analogo decreto per il figlio del papa ${ }^{46}$, anche se la sua riconosciuta nobiltà di natali e le sue altissime relazioni lo pongono su un piano superiore, il Privilegium Romanae Civilitatis di Montaigne, in apparenza magniloquente, è in realtà ripetitivo, talora impreciso e generico, o almeno tale appare nel raffronto con il parallelo testo elaborato dalle medesime istituzioni e in anni non lontani per Hubertus Goltzius.

Indizio di una evoluzione dei gruppi sociali e dei codici di valori a Roma è il riferimento al patriziato romano, presente per il nobile Montaigne e mancante per il Goltzius. L'espressione (cives patriciique Romani), ricorrente, ha una sua valenza storica valorizzata dalla $\mathrm{Mori}^{47}$ per illustrare una fase critica per il ceto nobiliare romano, tra

45 Cap. 74. Per il testo vd. infra.

46 Giacomo Buoncompagno (1548-1612), duca di Sora, marchese di Vignola e Governatore Generale di S. Chiesa, era ritenuto figlio del papa Gregorio XIII (morto nel 1585).

47 Mori, Tot reges, p. 96, 386, 390-392. 
1527 e $1590^{48}$ : dopo il sacco di Roma, trauma profondo per la città, induttore di crisi politica e socio-economica ${ }^{49}$, la nobiltà tenta di ricompattarsi mettendo in essere una serie di meccanismi di cooptazione, tra cui la civitas, meccanismi volti ad assimilare personalità notevoli non pertinenti al proprio casato e anche non romani di origine. Il senato municipale funge da organismo di potere di un'aristocrazia in crescita verso una definizione stabile, che si collega strumentalmente al passato delle origini romane spesso mediante la rifunzionalizzazione delle omonimie e l'impiego di elementi materiali indicati o millantati come pertinenti alla romanità più antica e comprovanti la vetustà del nomen. Questo per sostanziare un modello di status in potenziale autonomia (e antagonismo) dall'autorità papale che dalla crisi esce e si rafforza in senso teocratico, con l'affermazione del primato religioso ${ }^{50}$; ma tale identità fortemente laica incide scarsamente sulla realtà politica e istituzionale pontificia e spesso pare integrarsi senza apparenti traumi.

Dal momento che i cives veri e propri hanno diritto elettorale attivo e passivo e sono essi che determinano la realtà civica mediante l'accesso alle magistrature, la nobiltà nel tempo viene a connettersi intrinsecamente alla civitas e viceversa. Così tra XVI e XVII secolo aumenta sensibilmente il numero di coloro che chiedono contestualmente cittadinanza e patriziato ${ }^{51}$; e, mentre decadono richieste e assegnazioni della semplice cittadinanza, si giunge al potenziamento estremo del prestigio della nobiltà legata ai titoli e alle concessioni sovrane ${ }^{52}$, conseguente alla «serrata» del ceto compiutasi nel corso dell'intero secolo XVI. Progressivamente sarà il diploma di patriziato, che seguiva al senatoconsulto e come già quello di civitas veniva spedito all'assegnatario a pagamento anche anni dopo il decreto, a divenire il più richiesto e attribuito ai forestieri, oltre che il più costoso ${ }^{53}$.

48 Mori, Tot reges, p. 381.

49 Il colpo è terribile e inatteso, anche perché inferto dall'imperatore Carlo V d'Asburgo, tutore e conclamato erede dello spirito imperiale romano oltre che difensore della cristianità, per il suo contrasto con il pontefice Clemente VII alleato di Francesco I re di Francia, ovvero con la politica papale tendente all'indipendenza dall'Impero. Il sacco di Roma infrange il mito della sua intangibilità e incrina il modello della saldatura ideale tra cultura umanistica, mito di Roma e potere imperiale-pontificio: A. GiardinA, A. VAuchez, Il mito, p. 83-85 e p. 115, nn. 21-22.

50 Con il Concilio di Trento cui si affianca una importante politica di splendore monumentale, pregna di simbolismi, a partire da papa Paolo III Farnese (1534-49): A. Giardina, A. VAUCHEZ, Il mito, p. 91.

51 MORI, Tot reges, p. 390-392.

52 Mori, Tot reges, p. 387.

53 Con tale diploma, di cartapecora e d'oro, si assegnava il titolo di Cittadini Nobili dell'Ordine dei Patrizi Romani; minore richiesta, pur nel riscontro, per il titolo di Cittadini Nobili Romani: MoRI, Tot reges, p. 392. 


\section{L'istituzione, le minute dei privilegia civilitatis, le suppliche. Montaigne, Goltzius e la chiesa di Roma}

A Roma, presso l'Archivio Storico Capitolino, nei Credenzoni della Camera Capitolina e ripartiti in tomi, si conservano i registri delle minute dei privilegia, per la maggior parte civilitatis, concessi dalla municipalità romana. La civitas poteva essere attribuita per decreto agli stranieri già residenti che presentassero richiesta scritta, generalmente per motivi pratici; tale cittadinanza va distinta dalla civitas onoraria, assegnata dalla Città a personaggi di riconosciuta rilevanza per ruolo sociale, valore personale, relazioni, ascendenze. Nel XVIII secolo i dati relativi a tale documentazione d'archivio sono stati classificati e registrati dal Magni ${ }^{54}$ negli Indici Generali, per facilitarne la consultazione.

A un esame cursorio delle concessioni di civitas per il XVI secolo, si osserva subito il notevole numero di decreti registrati ${ }^{55}$, pur non risultando sempre agevole la distinzione tra beneficiari italiani e stranieri, dal momento che spesso i nominativi sono riprodotti in forma italianizzata fin nell'originale e non è talora agevole evincere località di nascita o provenienze degli interessati se non altrimenti noti; a ogni modo, e prevedibilmente, si può rilevare una netta prevalenza degli assegnatari italiani, soprattutto dal centro-nord ${ }^{56}$. Il riscontro autoptico con le minute dei privilegi di pugno degli scribi mostra nella maggioranza trascrizioni frettolose di privilegia civilitatis in forma abbreviata, talora anche molto scarna, generalmente a firma di uno scriba solo ${ }^{57}$ o anche apparentemente non firmate, presumendosi l'ultima firma valida per i privilegi successivi ${ }^{58}$; dopo la registrazione dei nominativi dei tre Consoli/Conservatori,

54 Filippo Maria Magni, archivista, a partire dal 1736 cura i Rubricelloni con l'Inventario dell'Archivio della Camera Capitolina.

55 A titolo di esempio, dagli anni dal 1560 al 1588, i decreti registrati dal Magni risultano nel numero complessivo di 567, con concentrazione particolare in alcuni anni (1560: 52 decreti; 1561: 31 d.; 1562: 18 d.; 1563: 25 d.; 1564: 13 d.; 1565: 7 d.; 1566: 11 d.; 1567: 21 d.; 1568: 6 d.; 1569: 11 d.; 1570: 18 d.; 1571: 17 d.; 1572: 28 d.; 1573: 28 d.; 1574: 13 d.; 1575: 13 d.; 1576: 21 d.; 1577: 9 d.; 1578: 14 d.; 1579: 24 d.; 1580: 20 d.; 1581: 15 d.; 1582: 16 d.; 1583: 19 d.; 1584: 22 d.; 1585: 14 d.; 1586: 35 d.; 1587: 28 d.; 1588: 38 d.). La Mori (1996, 388), prendendo in esame l'intera documentazione dal 1560 al 1644, rileva come in una prima fase, fino al 1608, le concessioni di cittadinanza superino complessivamente il migliaio, mentre nel secondo periodo ne risultano solo 201. Evidentemente il ceto nobiliare, dei cives veri e propri, si è progressivamente ricompattato e chiuso all'ingresso di ulteriori elementi «stranieri».

56 P. Pavan, "Cives origine vel privilegio", in Il Campidoglio e Sisto V, a cura di L. Spezzaferro e M.L. TitToni, Roma, 1991, p. 37-41, part. p. 38-9.

57 Con qualche parziale eccezione, come per Horatius Fuscus e Vincentius Martholus, spesso firmatari entrambi, o anche per Julius Horologius e Horatius Fuscus.

58 Sulle mansioni dello scribasenato vd. Pavan, Cives, p. 38 e 40 n. 18; tra le prerogative ufficiali dello scribasenato negli Statuta Almae Urbis authoritate Gregori XIII a Senatu Populoque Romano edita et reformata, (Romae, 1611, 1. III, cap. 9, 137 s.) è, ovviamente, 
in questi decreti ci si limita a registrare tra i cives i personaggi indicati. Ci sono però anche privilegia riprodotti in forma ampia e in modalità articolate e talora complesse, impreziositi da ampi formulari denotanti pur nella stereotipia una ricchezza tipologica, con varianti peculiari. Tali divergenze trovano presumibile spiegazione nelle identità ovvero nella rilevanza sociale dei destinatari. I decreti più ampi e dettagliati sono difatti indirizzati a personaggi del mondo della cultura, dell'arte o legati agli ambienti del potere laico e religioso ${ }^{59}$. I decreti più scarni devono invece rivolgersi a destinatari considerati di minore rilevanza innanzitutto dagli scribi; potrebbe trattarsi di personaggi meno abbienti, ma, per quanto ridotta sia la registrazione, è certo che essi non possono presumersi nullatenenti. Gli stranieri che richiedono la cittadinanza sono già residenti ed evidentemente interessati a fruire almeno di parte dei benefici dei cives; per essi le condizioni prescritte sine qua non sono: l'abitazione in città, il possesso di una vinea entro cinque miglia da Roma, la permanenza nella capitale per i tre quarti dell'anno con la famiglia ovvero l'ubicazione cittadina delle attività lavorative. In questo caso, la cittadinanza consiste nel riconoscimento di uno stato di fatto, la residenza e anzi il radicamento in città. Essa dimostra una integrazione avvenuta, assimilando di fatto stranieri residenti spesso già da più di una generazione, e cives nati ${ }^{60}$; e del resto all'interno di entrambe le categorie una consistente maggioranza non godeva dei pieni diritti. Difatti per gli stranieri residenti cives facti non si prevedeva la cittadinanza piena, con diritto elettorale attivo e dunque la possibilità di ricoprire cariche pubbliche nel governo cittadino; tale esercizio del potere doveva restringersi alla nobiltà e ad essi non veniva

«privilegia eorum qui Cives Romani a Magistratu vel Populo creantur, et in cives assumuntur notare et publicare».

59 Sulla questione vd. Pavan, Cives, p. 39. Tra i numerosi decreti per notabili, trascritti nelle minute in forma ampia e curata, il Gregorovius (F. Gregorovius, "Alcuni cenni storici sulla cittadinanza romana", Reale Accademia dei Lincei, Classe di Scienze Morali 1 [1877], p. 1-35, part. 29 s.) valorizza e discute, tra gli altri, i privilegi del cardinale Ottone Truchsess di Augusta, amico dei Farnese, e di suo fratello (1560), del card. Cristoforo Madruzzi (1560), di Carlo Sigonio, autore tra l'altro di Fasti consulares (1560), di Pirro Ligorio (1560), di Paolo Manuzio fratello di Aldo (1561), del cardinale Carlo Borromeo (1563). Rari i decreti per le donne: Maddalena de' Medici, sorella del Papa ; Claudia Rangona (p. 139v-140r), che acquista visibilità attraverso il nobile padre da cui parrebbe derivarle anche il nome; egli garantisce per lei anche presentando i propri meriti gentilizi. Nel tempo i decreti di cittadinanza nelle minute diventano molto meno curati e più scarni, mentre le differenze si appianano; le firme degli scribi scompaiono anche dai pochi decreti più ampi, fino al 1609 (ultimo decreto raccolto nel t. I del Cred. I), dal 1593, data dell'ultima firma per Horatius Fuscus e Vincentius Martholus entrambi «scriba sacri SPQR».

60 S. FECI, "Cambiare città, cambiare norme, cambiare le norme. Circolazione di uomini e donne e trasformazione delle regole in antico regime", in A. ARRU, F. RAMELLA (a cura di), L'Italia delle migrazioni interne. Donne, uomini, mobilità in età moderna e contemporanea, Roma, 2003, p. 3-31, part. 12-14. 
concesso più di un attestato ${ }^{61}$. Precedentemente incolae urbis ma non cives $^{62}$, potevano trasmettere la civitas conseguita dietro loro richiesta a figli e discendenti con diritti connessi, che restavano parziali ${ }^{63}$.

I requisiti di residenza, possesso di immobili e altro, non sono però richiesti per la categoria dei cittadini onorari, gli illustri personaggi che conseguono la cittadinanza per meriti e per istanza di altri stimati viri, come è tra l'altro il caso di Goltzius e Montaigne; la procedura può anche essere condotta a termine mediante la semplice approvazione del Consiglio Pubblico ${ }^{64}$. Il destinatario onorario riceveva il diploma vero e proprio, trascritto in forma completa su una pergamena inviatagli a sue spese anche dopo anni ${ }^{65}$, a differenza dei comuni cives che dovevano presentarsi a Roma in Consiglio. Dunque nella grande categoria della civitas concessa agli stranieri, almeno due tipologie di cittadinanza risultano distinguibili ed esse non comportano analoghi diritti e doveri in rapporto alla partecipazione e alla gestione della respublica. Ai non illustri, interessati alla civitas per motivi pratici (iscrizione dei figli al seminario o altro) venivano talora concessi attestati su richiesta (le «fedi»), ma comunque essi erano esclusi dalla cittadinanza con privilegio, che poteva consentire l'accesso all'ordine senatorio e il diritto elettorale attivo.

Nelle minute alcuni elementi convenzionali si ripropongono stereotipati e abbreviati a vari livelli: ad esempio, in apertura, la sigla Pri: ro: (o, per esteso, privilegium civilitatis ro:) + obtentum per I./Ill. + nome del civis (generalmente in accusativo),

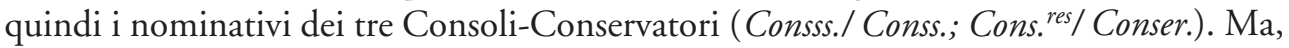

61 La Mori (Tot reges, p. 388-390) osserva come, tra il 1560 e il 1608, più della metà dei privilegi di cittadinanza siano stati concessi a personaggi di cui si premette la nobiltà della famiglia (come per il Montaigne), in misura molto minore la concessione è motivata da virtù intellettuali e meriti culturali (è il caso del Goltzius) o da particolari relazioni con la Curia Pontificia. A tutti costoro, dietro loro richiesta o per iniziativa degli organismi preposti, veniva attribuita la cittadinanza di pieno diritto. I restanti, non rinomati per meriti culturali o altro, mai sarebbero entrati nel Consiglio perché preventivamente sottoposti a rigidi criteri di selezione.

62 Pavan, Cives, p. 37: incola, che non è sinonimo di civis (ex origine vel privilegio), indica lo straniero che dimora a Roma, vi esercita un'attività, vi possiede beni stabili, ma non accede ai pubblici uffici e alle magistrature deliberanti; ad esempio (ibid., p. 38) non può sedere nel Consiglio pubblico e, se registrato come cittadino, non prima di un quinquennio; non può essere nominato scriba senatus, ufficio per il quale si deve essere cives originarii ex patre cive originario e incensurato.

63 La cittadinanza acquisita, dunque, non comportava in tutti i casi il pieno godimento dei diritti civici. Come si vedrà anche più oltre, l'oligarchia municipale non avrebbe potuto concedere indistintamente a tutti i nuovi cives il diritto elettorale attivo.

64 Pavan, Cives, p. 37.

65 Pavan, Cives, p. 38. Da documentazione d'archivio, la Mori (Tot reges, p. 392) ricava l'esistenza, nel primo Settecento, di tre tipologie di cittadinanza su diplomi di corrispondente ricercatezza nei materiali e nella fattura; due su tre sono destinate a nobili. 
raffrontando le minute dei nostri due decreti ${ }^{66}$, c'è un elemento che immediatamente colpisce: la differente lunghezza delle due stesure manuali a confronto con i testi pubblicati, che risultano invece di ampiezza sostanzialmente analoga (quindici - sedici righe). La minuta del privilegio del Montaigne mostra un testo estremamente abbreviato, di poco più di cinque righe in tutto inclusa l'intestazione; esso è integrato in un'unica pagina insieme ad altri quattro decreti ugualmente brevi e presumibilmente destinati a personaggi non rilevanti. Michaelis Montagna (nel decreto pubblicato Michaelis Montanus) vi è presentato con i suoi titoli, cui seguono i nominativi dei Conservatori, un primo segno di abbreviazione e la sezione iniziale della premessa, da «cum veteri more» a «suscepti sint» con un secondo segno di abbreviazione (per sottintendere il seguito), senza data né firma degli scribi. Anche nella minuta del Goltzius manca la firma degli scribi (ma sono registrati luogo e data). Tuttavia il decreto per Hubertus Goltzius occupa lo spazio di una intera pagina del registro di minute distinguendosi nettamente dai vicini privilegia, e inoltre riproduce abbastanza fedelmente il testo pubblicato, fatta eccezione per qualche rara abbreviazione. Non c'è confronto: tale minuta, vicinissima all'originale pubblicato come non quella del Montagne, è riprodotta dal trascrittore, altrimenti frettoloso e approssimativo, con l'attenzione e la cura destinate ai privilegia civilitatis dei non-romani di riconosciuta rilevanza culturale e sociale.

Ancora, mettendo a confronto i formulari delle due premesse (nella minuta del Montaigne manca tutto il restante) con le altre minute affini nel registro, sempre per il secolo XVI, si può osservare che per il decreto di Michel de Montaigne esistono riscontri molteplici, sia ampi che parziali e a differenti livelli, anche minimi; e che dunque la tipologia adottata per il decreto, quella di cui il Montaigne va tanto fiero, fa riferimento in realtà a un modulo assai comune, variamente ripreso e abbreviato. Sono infatti davvero numerosissimi i decreti di civilitas, ora più curati ora essenziali, introdotti dalla clausola Quod + nominativi dei Consoli + per Ill. ${ }^{\text {mum }}$ [nella minuta] oppure de e ablat. del nome del civis [nel testo pubblicato] + (Romana) civitate donando ad senatum

66 Goltzius: Registro di Privilegi di Cittadini Romani Creati, Cred. I, tomo 1, $91 \mathrm{v}-92$ r; Montaigne: ibidem, Cred. I, tomo 1, 154 v). Il Magni riporta per il privilegio del Montaigne la data del 1580, ma è evidentemente un suo errore, in quanto l'anno 1581 è confermato dalla supplica dello stesso Montaigne e dalla registrazione della sua nomina per il 13 marzo 1581 (volume dei Decreti di consiglio, Cred. I Tomo 28, sempre all'Archivio Storico Capitolino, a 59 v, 13 marzo 1581, tra i cives "nominati» [nominati vero pro futura civilitate ossequenda... ] cui è aggiunta la nota qui omnes supranominati tamque Ill.mi etiam cives ro. creati fuerunt). Nel Registro dei privilegi (Cred. I. tomo I, $154 \mathrm{v}$ ) si osserva che il privilegio precedente che è del 1580 e che il privilegio di Montaigne risulta senza data; è dunque possibile che il Magni si sia confuso. Il privilegio successivo, di Pietro e Hieronimo Victricius, reca la data III Idus Martii 1581; del resto, nel volume dei Decreti, i Victricii risultano cittadini creati insieme al Montaigne. Desidero ringraziare anche per tali rilievi le dottoresse dell'Archivio Storico Capitolino Cristina Falcucci ed Elisabetta Mori. 
retulerunt S.P.Q.R. de ea re ita fieri censuit (variamente abbreviato, fino a $D E R I F C$ ) ${ }^{67}$; in verità nella minuta quest'ultima parte manca e in sua vece compare il primo segno di abbreviazione seguito da «incipienti privil. ${ }^{\circ}$ ut sequitum». Anche per la formula successiva, "Cum veteri more et instituto cupide illi semper etc. [...]" esistono numerosi riscontri nelle minute, in abbreviazione e non ${ }^{68}$, oltre a casi più particolari perché rivolti ad assegnatari illustri, come i decreti, a firma degli stessi scribae Fuscus e Martholus, per Octavius Caesarinus Nolanus, per Michailis Mircatus o per Cosmus di Midicis (Cosimo de' Medici) Francisci f. ${ }^{69}$, del 1579 e del 1580, decreti quasi analoghi nell'impianto generale e nelle formule, salvo le indicazioni personali, a quello pubblicato dal Montaigne negli Essais. Anche la precisa formula relativa ai privilegi e agli onori di cui il civis può godere si riscontra altrove ${ }^{70}$. $\mathrm{E}$ dal momento che nella minuta il decreto per il Montaigne ha termine subito dopo la seconda abbreviazione, è evidente che quanto manca del testo poteva dirsi scontato e anche che lo scriba doveva sentirsi autorizzato all'intervento riduttore e standardizzante senza particolari difficoltà.

Per la formula introduttiva del senatoconsulto di Goltzius, con i nomi dei consules senza il quod + ad posteritatis monumentum [...] omnium nationum res gestas superaret, invece, a un controllo delle minute non si rinvengono precisi riscontri. Proseguendo, per l'espressione relativa all'accrescimento della respublica romana («augendae reipubl. gratia») come finalità della cittadinanza onoraria possono rinvenirsi confronti in molti decreti, ma mai in forma di letterale riproduzione, bensì nella formula fissa, già qui richiamata, «cum ad augendam» + segno di abbreviazione ${ }^{71}$ o più estesamente «cum ad augendam servandamque Rempublicam $[\ldots]^{72}$ ». L'elenco dettagliato delle prerogative che il nuovo civis può esercitare, nella modalità in cui sono esposte per Goltzius, trova invece riscontri diversi, ad esempio nel decreto per i fratelli Maior o per Julius

67 Registro di Privilegi di Cittadini Romani Creati, Cred. I, tomo 1, ad es. 55, 64, 65 r-v, 68 v, 70 r, parzialmente 90 v, 91 v, 92 v, 93 r-v, 94 r-v, 95 r-v, 152 r-v, 153, 162, passim.

68 Registro di Privilegi di Cittadini Romani Creati, Cred. I, tomo 1, ad es. 90 r-v, 151 e passim; tra gli esempi più dettagliati (oltre a quelli indicati nella nota successiva) possono considerarsi i decreti per Aurelius Portulaca (1561), 69; quello per Petrus et Aloysius Maior (1561), 65 r-v e altri (passim). Su due pagine affrontate Julius Horologius cominciava un decreto con "cum ad augendam servandamque rempublicam» e l'altro con "cum veteri more et instituto»; dunque il formulario è fisso, impiegato negli stessi anni, e viene alternato a discrezione per variatio.

69 Registro di Privilegi di Cittadini Romani Creati, Cred. I, tomo 1, 150 r-v, 151, $152 \mathrm{v}-153 \mathrm{r}$.

70 Ad es. nel Registro di Privilegi di Cittadini Romani Creati, Cred. I, tomo 1, $151 \mathrm{r}$.

71 Registro di Privilegi di Cittadini Romani Creati, Cred. I, tomo 1, ad es. 92 r-v, 93 r-v, 94 r-v, 95 r, 96, 97 v., 150, 152 r-v, 153, 154 r-v, 155, 156, 189, 190, passim. Tra fine XVI e inizio XVII secolo si assiste nei registri delle minute a una prevalenza della schematizzazione minima dei privilegia Romanae Civilitatis, mentre i decreti «maggiori», dettagliati e curati, finiscono per scomparire.

72 Registro di Privilegi di Cittadini Romani Creati, Cred. I, tomo 1, 97 r. 
Jacobonius ${ }^{73}$; tale formula, che prevede tra l'altro un ruolo attivo del civis nelle magistrature laiche e religiose o, meglio ancora, la sua applicazione (che resti potenziale o si traduca in pratica politica), deve considerarsi intrinsecamente connessa con l'esercizio esclusivo del potere politico da parte della nobiltà romana ${ }^{74}$. L'espressione successiva, sulla claritas dell' istituzione e sulle sue finalità, è parafrasata, variata e adattata per il Goltzius su un formulario di base, come prova il raffronto con qualche altro decreto, sempre tra quelli più dettagliati, che presenta locuzioni similari, comunque non analoghe ${ }^{75}$; e che ci sia una consapevolezza nelle opzioni e non si tratti di una evoluzione diacronica di moduli standardizzati lo dimostra il raffronto con un decreto del 1561, precedente anche al privilegio di Goltzius, in cui si riproducono formule in tutto analoghe a quelle del decreto del Montaigne di quattordici anni dopo, per personaggi tra l'altro dalle qualifiche in parte affini (Petrus e Aloysius Maior a cubiculo Serenissimi Ferdinandi Archiducis Austriae [...] ${ }^{76}$.

Il testo approntato per Goltzius risulta così più originale e accurato anche sotto il profilo stilistico.

Dalla documentazione superstite è riscontrabile la prassi secondo cui chi desiderava ottenere la civitas romana poteva presentare una supplica, sorta di richiesta ufficiale, ai Conservatori, da ratificarsi nel Consiglio segreto. Un registro che raccoglie tali documenti è attualmente visionabile presso l'Archivio Capitolino ${ }^{77}$. Al controllo, si è potuta individuare la supplica del Montaigne, che potrebbe essere stata scritta di suo pugno dal momento che egli conosceva la lingua italiana ${ }^{78}$; di essa segue trascrizione. Non è stato invece possibile identificare la supplica di Hubertus Goltzius (Uberto Goltzi, così italianizzato dal Magni) che parrebbe mancare.

73 Registro di Privilegi di Cittadini Romani Creati, Cred. I, tomo 1, 67, fratelli Maior (1561); 151, Julius Jacobonius (1580). Giulio Jacobonio era un noto erudito, autore, tra l'altro, dell'operetta Ad Marcellum Caroccium in Horatii gemini fratris obitu Julii Jacobonii consolatio (1580), su modello delle consolationes classiche, e di un'appendice all'opera di Joannes Baptista Fonteius, De prisca Caesiorum gente, commentariorum libri duo (1583).

74 Su cui vd. Mori, Tot reges, p. 391.

75 Ad es., ancora nel Registro di Privilegi di Cittadini Romani Creati, Cred. I, tomo 1, il citato Privilegium Romanae Civitatis per Petrus e Aloysius Maior del 1561, trascritto e firmato ugualmente da Julius Horologius.

76 Vd. n. prec.

77 Memoriali de' Cittadini Romani Creati, Cred. IV, tomo 64, 176.

78 Michel de Montaigne, educato dal padre all'italianismo' secondo la tendenza francese dell'epoca leggeva, ben comprendeva, scriveva o/e trascriveva la lingua italiana: vd. F. Garavini, "Sull'italiano del Journal de Voyage", in Ead., Itinerari a Montaigne, Firenze, 1983, p. 119-133; F. Caldari BevilacQua, "Montaigne alla Biblioteca vaticana", in Montaigne e l'Italia, Atti del Congresso Internazionale (Milano-Lecco, 26-30 ottobre 1988), Torino-Genève, 1988, p. 363-390, part. 366-7; C. CAvallini, L'italianisme de Michel de Montaigne, Paris, 2003, p. 10-11, 15-17, 23 ss., passim. 
"Ill" : Sig ${ }^{r i}:$

Desiderando da V.S. Ill ${ }^{m e}$ : et da codesto inclito Popolo essere aggregato nel numero delli altri loro Cittadini, vengo a supplicarle che si degnino conumerare me, et miei figlioli, fra loro Cittadini con concedermi li soliti privileggi, che tutto ripporterò per grazia singolare, offerendomi a VV. SS. Il ${ }^{m e}$ : et a codesto inclito Popolo espore la propria vita in loro servitio di casa. Il di XII di marzo 1581 [espressione non chiara, forse così decodificabile]

Di VV. SS. Ill ${ }^{\text {me }}$ : Servitore

Michele di Montagna cavallier de l'ordine del re christianissimo \& gentiluomo ordinario de la su camera».

Nel Viaggio in Italia ${ }^{79}$ il Montaigne racconta che nel corso del suo soggiorno a Roma, nel marzo 1581, cercò di ottenere il titolo di civis, ma incontrò delle difficoltà superate solo grazie a un intervento molto autorevole. Ecco quanto egli riferisce testualmente:

"Je cercherai pourtant et employai tous mes cinq sens de nature pour obtenir le titre de citoyen romain, ne fut-ce que pour l'ancien honneur et réligieuse mémoire de son autorité. J'y trovai de la difficulté; toutefois je la surmontai, n'y ayant employé nulle faveur, voire ni la science seulement d'aucun Français. L'autorité du pape y fut employée par le moyen de Filippo Musotti, son maggiordomo, qui m'avait pris en singulière amitié et s'y peina fort. Et m'en fut dépêché lettres $3^{\circ}$ Id. Martii $[13$ marzo]1581, qui me furent rendues le 5 avril très authentiques, en la même forme et faveur de paroles que les avait eues le seigneur Jacopo Buoncompagno, duc de Sora, fils du pape. C'est un titre vain; tant y a que j'ai reçu beaucoup de plaisir de l'avoir obtenu».

«Mi sono adoperato quindi, e ho impiegato tutti i miei cinque sensi naturali, per ottenere il titolo di cittadino romano, non fosse altro che per l'antico onore e la sacra memoria del suo prestigio. Vi ho incontrato difficoltà; tuttavia le ho superate senza aver impiegato alcun favore, neppure la semplice conoscenza di qualche connazionale. Vi concorse l'autorità del papa, per il tramite di Filippo Musotti suo maggiordomo che mi aveva preso in particolare amicizia e si impegnò molto. E nel terzo giorno dalle Idi di marzo [13 marzo] 1581 me ne fu spedita la bolla che mi giunse il 5 aprile autenticissima, nella stessa forma e con espressioni altrettanto lusinghiere di quelle che aveva ricevuto il signor Jacopo Buoncompagno, duca di Sora, figlio del papa. È un titolo senza utilità; eppure ho provato molto piacere nell'averlo ottenuto».

79 Journal de Voyage en Italie par la Suisse et l'Allemagne en 1580 et 1581. Originariamente, il testo, per gran parte compilato da un famiglio di Michel de Montaigne, non era destinato alla diffusione; rinvenuto nel castello di Montaigne dall'abate de Prunis, venne pubblicato nel 1774 da Meusnier de Querlon. Su Montaigne nel suo rapporto con l'Italia: E. Terlizzi, Montaigne e l'Italia, Napoli, 1921; nell'opera miscellanea Montaigne e l'Italia, si vedano in particolare i saggi di M. Simonin, M. Tetel, M. Dal Corso, F. Charpentier, M. Bideaux, R. Chevallier, Ch. Béné. 
Non tutti gli stranieri illustri insigniti della cittadinanza onoraria presentavano una supplica. In un suo studio sulla cittadinanza romana, la $\operatorname{Pavan}^{80}$ ha osservato che dal 1580 al 1595 a fronte della concessione di 580 cittadinanze (ma il privilegio risulta spedito solo per 321 di esse ${ }^{81}$ ) restano 465 suppliche originali. Effettuando un più ampio confronto tra i registri delle suppliche e i verbali del Consiglio, la studiosa ha potuto concludere che risultano mancanti senz'altro le suppliche relative a cittadinanze onorarie proposte direttamente dal Senatore o dal Papa, per le quali non era indispensabile il rispetto dell'ordinaria procedura. A tale proposito, in verità, la Mori ${ }^{82}$ valorizza anche il dato apparentemente divergente delle 280 suppliche dal 1585 al 1587 di contro alle 70 registrazioni di cittadinanza, osservando per questi anni il numero maggiore di suppliche rispetto alle concessioni di civitas ovvero la sostanziale discontinuità dei due ordini di documenti, fondati su differenti logiche e richiedenti valutazioni specifiche per problematiche e ordini di confronto. Il fenomeno osservato dalla Pavan potrebbe per il nostro caso contribuire a spiegare il motivo per cui la supplica di Hubertus Goltzius non risulta in archivio: il personaggio è universalmente noto, apprezzato presso le corti d'Europa e nei circoli culturali più rilevanti proprio per i suoi contributi di studio e di ricerca su Roma antica; peraltro in suo favore nel privilegio si richiama la testimonianza praeclarissimorum civium ed è possibile che l'onorificenza gli sia stata offerta senza alcuna formale richiesta. Viceversa, la medesima considerazione getta un'ombra inquietante sul motivo della supplica inoltrata invece dal Montaigne, che scrive di aver tentato in ogni modo di conseguire la cittadinanza di Roma; per lui, a suo stesso dire, si interessò il Papa in persona e dunque della supplica non si ravviserebbe necessità. Dalla documentazione e dalle considerazioni della Mori, emerge per la nobiltà romana la chiara tendenza, in progressivo aumento per tutto il secolo ma con ritmo non costante e più evidente nell'ultimo ventennio, a ridurre le nuove assegnazioni di cittadinanza a fronte di una richiesta che tende invece ad aumentare: ricostruitasi dopo il 1527 in costante dialettica di incontro/scontro con il potere pontificio, dopo una iniziale fase di apertura alla civitas onoraria per i non-romani, la nuova nobilitas ricostituita tenta a più riprese di limitare potenziali ingerenze esterne con ulteriori ingressi nel corpo civico.

A quanto lui stesso racconta, per acquisire la civitas romana il Montaigne non fa ricorso all'aiuto di connazionali. Eppure nel Cinquecento a Roma i Francesi costituivano per consistenza la seconda «nazione ${ }^{83}$, e nel corso del suo soggiorno lo stesso Montaigne si mostra in rapporti di amicizia con personalità francesi di rilievo ${ }^{84}$ : con l'ambasciatore di Francia a Roma, conte d'Albain, nobile studioso e suo amico da

80 Pavan, Cives, p. 38.

81 Forse perché la spedizione e anche il costo del diploma vero e proprio erano a carico dell'assegnatario e inoltre perché non tutte le cittadinanze concesse erano onorarie.

82 Mori, Tot reges, p. 388.

83 A. Esposito, "La città e i suoi abitanti", in A. Pinelli (a cura di), Roma del Rinascimento, Roma-Bari, 2001, p. 3-47, part. 6.

84 Journal de Voyage, cap. 72 (ROMA: 30 novembre 1580 - 15 marzo 1581). 
tempo, che gli consiglia di recarsi in udienza dal Pontefice per il «bacio della pantofola» (29 dicembre 1580); con Charles d'Estissac ${ }^{85}$, che lo accompagna pure alla stessa udienza papale cui sono presenti anche altri francesi, i signori de Mattecoulon e du Hautoi; con Nicolas de Pellevé cardinale de Sens, presso cui il Montaigne resta a cena l'ultimo di dicembre (peraltro il de Sens è nella congregazione dell'Indice incaricata di valutare gli Essais $^{86}$ ); con l'erudito Muret e con altri ancora ${ }^{87}$. Il papa, Gregorio XIII, ha parole di incoraggiamento per lo studio e la virtù del d'Estissac, e prega il Montaigne di mantenere la devozione sempre manifestata per la Chiesa «e per il servizio del re cristianissimo", dichiarando al commiato la propria disponibilità personale (ma l'espressione viene interpretata come puramente convenzionale: «Si tratta di modi di dire italiani»).

Tali appoggi non avrebbero evidentemente comportato, e forse non comportarono, i risultati sperati per il conseguimento della onorificenza. Doveva essere necessario il più autorevole sostegno, quello del Pontefice in persona; e il Montaigne riesce a ottenerlo, sempre a quanto egli racconta, non grazie alla benevolenza precedentemente manifestata da Gregorio XIII nei suoi confronti, ma per l'intervento del maggiordomo del papa, Filippo Musotti ${ }^{88}$, con cui il Francese aveva instaurato un buon rapporto di amicizia. E ciò nonostante, il Montaigne presenta anche una supplica con richiesta formale di cittadinanza romana e chiarissima professione di umiltà.

Si potrebbe concludere, e per altri versi la considerazione è stata a suo tempo espressa dal Gregorovius ${ }^{89}$, che per la municipalità romana il Montaigne non doveva avere credenziali tali da meritare per sé stesso la cittadinanza onoraria. Egli la consegue non tanto per le sue personali insistenze (i suoi «cinque sensi» attivati allo scopo), quanto soprattutto per l'appoggio del Papa, il cui intervento tuttavia parrebbe dovuto più alla stima per il suo maggiordomo che per l'intellettuale francese. D'altra parte, a

85 Sul nobile francese, studioso ed erudito, Carlo d'Estissac vd. L.-A. Colliard, "Un jeune ami de Montaigne: Charles d'Estissac”, in G. Bernardelli, E. Galassi (a cura di), Lingua, cultura e testo. Miscellanea di studi francesi in onore di Sergio Cigada, Milano, 2003, p. 273-284, part. 273-276. Egli fa parte del gruppo di accompagnatori francesi nel viaggio e reca lettera di raccomandazione del re Enrico III e della regina madre Caterina al duca di Ferrara e a papa Gregorio XIII (E. CAMESASCa [a cura di], Michel de Montaigne, Viaggio in Italia, Milano, 2003, p. 515).

86 Caldari BevilacQua, Montaigne, p. 366.

87 Journal de Voyage, cap. 72: si menzionano anche i signori du Bellai, il barone de Chasai e il de Marivau con altri personaggi.

88 Già segretario del cardinal Seripando, quindi del cardinale di Lorena, il Musotti è anche noto per aver raccolto sotto il titolo di Giornale del Concilio di Trento un gran numero di lettere originali: vd. P. SARPI, Istoria del Concilio Tridentino, IV, Firenze, 1858, "Appendice" (a cura di P.F. COURAYER), XXII. In verità la Caldari Bevilacqua (Montaigne, p. 366) ritiene possibile una confusione con il fratello di Filippo, Alessandro Musotti, maggiordomo del papa, salvo poi ammettere la plausibilità del riferimento.

89 Gregorovius, Cittadinanza, p. 30. 
quanto leggiamo ancora dal Journal de Voyage ${ }^{90}$, giunto a Roma nel dicembre 1580, il Montaigne subisce un'accuratissima perquisizione dei bagagli e il sequestro di tutti i suoi libri per il vaglio degli organismi pontifici competenti, inclusa la copia personale degli Essais, opera peraltro munita di privilegio reale e di autorizzazione della curia di Bordeaux; viene subito chiarito che i testi non saranno presto restituiti. I criteri che a Roma si applicano per l'indagine censoria sembrano subito molto restrittivi e non facilmente comprensibili o condivisibili: la confisca di un testo di preghiere perché parigino e non romano e di opere tedesche anti-ereticali in quanto descrivono gli errori delle eresie sia pure per confutarli.

In un libro recente, Inquisitori, censori e filosofi sullo scenario della controriforma ${ }^{91}$, Saverio Ricci indaga tra l'altro sul rapporto tra Michel de Montaigne e la Chiesa di Roma, valorizzando la tappa romana del Voyage e mettendo in luce gli elementi di ambiguità nell'accoglienza ricevuta dall'ambiente ecclesiastico, apparentemente benevolo. Proprio le strette relazioni con Caterina de' Medici ed Enrico III di Valois, pur nella esortazione dello stesso Pontefice al Montaigne, a seguitare nella devozione alla Chiesa e al re di Francia, possono rappresentare un punctum dolens della questione, dal momento che i Valois, dopo San Bartolomeo, non perseverano nella lotta contro il protestantesimo, mentre a Roma si è in pieno Concilio Tridentino. Si può aggiungere a ciò la resistenza francese alla piena attuazione dei decreti tridentini e l'apertura agli eretici e all'Inghilterra da parte del duca di Anjou, fratello di Enrico III ${ }^{92}$. In Italia la lotta contro l'eresia sta invece comportando l'istituzione di un sistema censorio vieppiù capillare che confluirà negli Indici ${ }^{93}$, il cui obiettivo finale è il dominio sulla spiritualità stessa del mondo cattolico anche mediante la censura preventiva delle opere destinate alla pubblicazione e alla diffusione; il che implicherà come conseguenza una sorta di autocensura degli Autori stessi. Anche la devozione alla Chiesa da parte del Montaigne

90 Cap. 72.

91 S. Ricci, Inquisitori, censori, filosofi sullo scenario della Controriforma, Roma, 2008, p. 99-220.

92 Caldari Bevilacqua Montaigne, p. 363.

93 Dopo la pubblicazione dei primi Indici romani dei libri proibiti (1559, 1564), tre organismi sono istituiti per portare a compimento il programma totalizzante: il Santo Uffizio, che vigila sull'ortodossia; la Congregazione dell'Indice dei libri proibiti, che esercita la propria attività su tutto il mondo cattolico; il Maestro del Sacro Palazzo, il teologo del papa, a Roma responsabile della censura. Il programma prevede due fasi. La prima consiste nella messa all'Indice preventiva delle opere che per una qualsiasi ragione possono apparire sospette. La seconda prevede l'intervento vero e proprio sui testi che, se giudicati recuperabili, sono sottoposti alla emendatio. Lungi dal limitare l'intervento all'eresia teologica, la Chiesa tende ad estendere il proprio controllo a tutti i campi del sapere, dagli studi politici e giuridici alla fisica, alla scienza, alla matematica, all'astronomia, ai trattati di magia, alle arti esoteriche. L'attività censoria investe anche la letteratura, antica e moderna, inclusi i poemi cavallereschi, la poesia erotica, i romanzi; la maggiore imputata è però la filosofia (Giordano Bruno, Tommaso Campanella). 
può essere risultata di non piena credibilità all'ambiente ecclesiastico romano: egli aveva tradotto (1569) la Theologia Naturalis di Raymond Sebond senza considerare il divieto di lettura dell'opera ${ }^{94}$ e negli Essais elogiava Giorgio Buchanan e Theodoro de Bèze ${ }^{95}$, condannati dalla Chiesa per le loro traduzioni di testi biblici, dando più di un segno della propria libertà di pensiero, in Francia del resto apprezzata. Pur accostandosi spesso nelle proprie convinzioni alle posizioni della Chiesa cattolica, nella sua sostanziale a-religiosità ${ }^{96}$, il Montaigne mostra inoltre in più di un episodio concordanze ideologiche con il «rovinoso» Machiavelli e, ancora, una concezione di fortuna più simile all'idea degli antichi che all'ortodossia cattolica; senza considerare la parziale giustificazione di Giuliano l'Apostata.

Il lunedì della settimana di Pasqua del 1581, al Montaigne viene restituita la sua copia degli Essais, esaminata ed emendata da una commissione ecclesiastica nella quale un ruolo di rilievo aveva ricoperto padre Sisto Fabri, domenicano e Generale dell'Ordine, Maestro di Palazzo Apostolico, che si era però servito della relazione di un frate francese ${ }^{97}$, adottando le correzioni da questi indicate. Il Montaigne discute con lui ogni punto contestato riuscendo a limitare l'intervento censorio; anzi il Fabri rimette alla sua coscienza gli emendamenti da apporre all'opera, in contrasto a propria volta con altri di opinione opposta ${ }^{98}$. Alla partenza da Roma, il Francese viene così invitato a tener conto per le future edizioni degli Essais di una serie di suggerimenti e di correzioni che egli in realtà non avrebbe né considerato né tanto meno adottato; in seguito i censori sarebbero intervenuti a emendare a fondo la traduzione italiana, fino alla condanna degli Essais in tutte le lingue ${ }^{99}$.

94 L'opera riduce il ruolo mediatore della Chiesa nella esegesi delle Scritture, a favore della dimostrabilità razionale delle verità; tratta inoltre del libero arbitrio e dell'eccellenza dell'uomo. Negli Essays (II 12) può leggersene la difesa contro i detrattori (l'Apologia di Raymond Sebond: un'edizione italiana recente è quella curata da D. Fusaro, Milano, 2004); vd. anche TOMASI DI LAMPEDUSA, Lettere francesi, p. 105-107.

95 Essais I 26; II 17. Vd. D.B. Heitsch, Practising reform in Montaigne's Essais, LeidenBoston-Köln, 1968, p. 119; A. JoHnSON, Knowledge and society: a social epistemology of Montaigne's Essais, Charlottesvilles, Virginia, 1994, p. 134-5, 167-8 n. 8 (Montaigne-de Bèze); N. Panichi, I vincoli del disinganno. Per una nuova interpretazione di Montaigne, Firenze, 2004, p. 255.

96 Senza mai, però, una presa di posizione netta per l'ateismo. Anche dalla lettura del Catechismo dell'eretico italiano Bernardo Ochino, inizialmente rifiutata, il Montaigne scrive di aver tratto grande profitto: TOMASI DI LAMPEDUSA, Lettere francesi, p. 100, 105.

97 Per mancanza di conoscenza della lingua.

98 Journal de Voyage, cap. 74.

99 La censura pontificia sarebbe stata in qualche modo guidata dalla precedente doppia condanna, teologica e morale, degli Essais da parte delle autorità calviniste nel 1602; nel 1676 è decretata la condanna dell'opera del Montaigne in tutte le lingue. 
Il decreto di civitas per Hubertus Goltzius denota dunque di per sé un superiore apprezzamento da parte delle maggiori autorità municipali romane e anche dello Stato Pontificio ${ }^{100}$, pur apparentemente con evidenza minore. Le sue opere celebrano del resto, enfaticamente e in forma diversificata per contenuti e anche per tecniche di riproduzione editoriale, i fasti dell'impero e della romana respublica, consentendo nell'attualità la valorizzazione funzionale dei legami con il passato antico e più recente («il Comune antico»), in linea con l'ideologizzazione della Roma repubblicana propria della propaganda della nuova nobiltà, che fa riferimento al senato municipale. La ricostruzione identitaria del gruppo sociale, che include il «farsi romani» ossia una graduale assimilazione dei non-romani, si volge al culto della grandezza del passato, della respublica, come del resto seguitava a definirsi lo pseudo-governo cittadino insediato sul Campidoglio, tollerato o contrastato dai Pontefici, certo da essi progressivamente esautorato. La mitologizzazione delle origini perde nel tempo la carica polemica antipapale divenendo "pratica retorica congelata nella sua valenza antiquaria», «insegna araldica di un intero ceto priva di qualsiasi aggressività politica ${ }^{101}$ ».

Dunque, le politiche papali non solo non risultano incompatibili con i modelli repubblicani evocati; accomunate dal culto per l'antichità e dal collezionismo, esse diventano accondiscendenti, ove non favorevoli, nei confronti di elementi culturali potenzialmente antagonisti e tuttavia riducibili, con tacito accordo della controparte, a uno spessore puramente erudito rispondente alla logica della condivisione urbana del potere. Gli stranieri, i non-romani, cooptati nel corpo civico e nel ceto nobiliare, chiamati a condividere privilegi e doveri con i cittadini di pieno diritto, sono grati all'autorità romana che è innanzitutto papale, e si identificano nel modulo mitico e attuale insieme di Roma «madre di tutti i popoli».

Che le gerarchie ecclesiastiche di Roma, oltre agli ambienti laici, avessero accolto Goltzius con grande favore emerge anche da una scorsa ai nominativi di coloro che nell'Urbe gli aprono la porta delle proprie collezioni numismatiche e che egli ringrazia nella succitata lista dal C. Julius Caesar innanzitutto il pontefice Pio IV, papa dal 1559 al 1565, al secolo Giovanni Angelo Medici di Marignano, zio del cardinale Carlo Borromeo ${ }^{102}$, quindi nell'ordine cardinali, vescovi, canonici, laici. Leggiamo i nomi del cardinale Alessandro Farnese (il Giovane), vicecancelliere di Santa Romana Chiesa,

100 Si ricordi il richiamo agli iura del Pontefice da osservare in quanto civis.

101 MORI, Tot reges, p. 380.

102 A. Menniti Ippolito, Il governo dei papi, p. 62-64. 
nipote di Paolo III, diplomatico e negoziatore tra le maggiori potenze europee ${ }^{103}$, appassionato d'arte e grande collezionista di monete antiche e gioielli; del fratello Rainuzio Farnese, parimenti cardinale, penitenziario maggiore, mecenate di uomini di cultura e collezionista di antichità ${ }^{104}$; di Rodolfo Pio da Carpi ${ }^{105}$, cardinale influente e diplomatico, anch'egli mecenate e appassionato collezionista; del cardinale Federico Cesio ${ }^{106}$ della nobile famiglia dei Caesii; del cardinale mantovano Ercole Gonzaga ${ }^{107}$; del cardinale ferrarese Ippolito d'Este; del cardinale Giovanni Hieronimo Morone ${ }^{108}$; del cardinale milanese Carlo Borromeo ${ }^{109}$, dei cardinali fiorentini Thadeo de Gaddis

103 Nella preparazione della partenza dello stesso Paolo III per Nizza Alessandro Farnese (1545-1592) cerca di ricomporre la contesa tra Francia e Spagna, tra Francesco I e Carlo V. Quindi, dopo un contrasto (1551-1552), superato, con il papa Giulio II, egli si reca presso Enrico II di Francia per poi rientrare in Italia. Gli si devono gli Horti Farnesiani, splendidi giardini sulle rovine del Palatino (1574) e il completamento di una sezione di Palazzo Farnese, che diviene una sorta di museo delle collezioni d'arte di famiglia. Appassionato d'arte, si fa ritrarre da Tiziano come i grandi dell'epoca e nel 1579 acquista la Farnesina su progetto del banchiere Agostino Chigi, con dipinti di Raffaello, del Sodoma, di Giulio Romano.

104 Come Giovanni della Casa, Annibal Caro, il Sigonio e altri. Rainuzio, o Ranuccio, Farnese (1530-1565) è il principale sostenitore e fautore della scelta di Fulvio Orsini, discendente non legittimo della famiglia Orsini, come curatore della biblioteca di Palazzo Farnese dal 1558, al servizio del «Gran Cardinale» Alessandro. Grazie al lavoro dell’Orsini (su cui vd. infra, n. 114) la biblioteca si arricchisce di manoscritti e pubblicazioni di valore.

105 1500-1564. Nunzio apostolico in Francia nel 1530, ricopre incarichi diplomatici fino al 1535, anno in cui prende parte alle trattative per la pace tra Francesco I e Carlo V. Amante delle arti e mecenate, protegge gli artisti senza differenze di credo ed è noto anche come collezionista di statue, libri, codici e medaglie.

106 Federico Cesio (1500-1565), di famiglia nobile e cardinalizia, nominato cardinale da Paolo III; amico di Carlo Borromeo, Ignazio di Lodola, Filippo Neri.

107 1505-1563. Mantovano, nato da Francesco II Gonzaga e Isabella d'Este, vescovo di Mantova, poi cardinale, tra i presidenti del concilio di Trento.

108 Giovanni Gerolamo Morone (1509-1580), diplomatico, noto per la sua opera di mediazione nei confronti delle eresie luterane; per tale atteggiamento è sottoposto a processo sotto il papa Carafa, l'intransigente Paolo IV, scagionato quindi dal successore, Pio IV.

109 1538-1584. Considerato il massimo interprete dello spirito della Controriforma, Segretario di Stato a Roma, prende parte all'ultima fase del Concilio di Trento (1562-1563) interpretando il proprio ruolo alla luce del dettato tridentino, sulla base dei severi principi morali e della lotta contro le eresie. Con rigore riorganizza la struttura ecclesiastica del Milanese, impiegando anche il contributo, tra gli altri, dei gesuiti, cui assegna la gestione dei seminari e dei collegi fondati per educare una nuova classe dirigente laica ed ecclesiastica. 
e Giovanni de' Medici ${ }^{110}$; del cardinale aretino Innocenzo del Monte ${ }^{111}$; del cardinale Nicolao de Sermoneta ${ }^{112}$; dei cardinali napoletani Carlo e Alfonso Carafa ${ }^{113}$. Seguono numerosi altri nominativi di vescovi, arcivescovi, canonici, quindi di personalità del mondo della cultura e dell'arte (tra cui Fulvio Orsini ${ }^{114}$, Antonio Agustín, Benedetto

110 Giovanni di Cosimo I de' Medici, detto il Giovane (1543-1562): figlio secondogenito del Granduca di Toscana Cosimo I e di Eleonora di Toledo, destinato alla carriera ecclesiastica, è nominato cardinale da Pio IV nel 1560, ma muore presto di malaria.

111 Innocenzo Ciocchi del Monte (1532-1577). Di umili origini, è accolto e protetto dal cardinale Giovanni Maria del Monte, il futuro papa Giulio II, che lo fa adottare dal fratello maggiore e lo nomina "cardinal nepote» (istituzione che confligge con la trasmissione non genealogica del potere pontificio, innescando elementi di instabilità e di contrasto: A. PINELLI, "Introduzione", in Roma del Rinascimento, p. V-XL, part. XVI), affiancandogli un cardinale segretario. Caduto in disgrazia sotto il papa Pio IV, rientra nell'Urbe in seguito alla elezione di Gregorio XIII.

112 Niccolò Caetani da Sermoneta: 1526-1585, cardinale dal 1536.

113 Carlo Carafa (1517-1561): nipote di Giovanni Pietro Carafa divenuto papa come Paolo IV, per volontà dello zio diviene cardinal nepote e Segretario di Stato con esiti non felici, al punto che nel 1559 lo stesso Paolo IV lo sostituisce (come cardinal nepote) con Alfonso Carafa. Nel 1560 papa Pio IV arresta gli esponenti più in vista dei Carafa per gli abusi di potere sotto il suo predecessore; così Carlo e il fratello Giovanni Carafa vengono condannati a morte e giustiziati per strangolamento. Alfonso Carafa (1540-65) è pronipote del papa Paolo IV (Giovanni Pietro Carafa) in quanto figlio di un nipote, Antonio. Nel 1557 diviene cardinale e poi arcivescovo di Napoli; anch'egli viene arrestato, travolto dal disonore degli zii, dal papa Pio IV, ma poi rilasciato. Sulla figura e sul ruolo del «cardinal nepote» nella corte pontificia vd. Menniti Ippolito, Il governo dei papi, p. 121-125. Su Alfonso e Carlo Carafa, sui rapporti con Paolo IV e le relazioni con la corte pontificia si vd. R. DE MAIO, Alfonso Carafa, Cardinale di Napoli (1540-1565), Città del Vaticano, 1961.

114 1528-1600. Erudito, bibliofilo, collezionista. Dopo aver servito come bibliotecario Paolo III Farnese, curandone la biblioteca e le collezioni artistiche ed antiquarie, Fulvio Orsini lascia in eredità alla famiglia la sua personale raccolta di gemme e medaglie. Autore anche di scritti di iconografia antica (Imagines et elogia, 1570) e di numismatica (Familiae Romanae, 1577), per suo mandato tutti i libri di personale proprietà verranno a costituire la Biblioteca Vaticana Orsini. 
Egio $^{115}$, Michelangelo Buonarroti, Pirro Ligorio ${ }^{116}$, Annibal Caro ${ }^{117}$ ) comunque attivi per la corte pontificia; infine i nominativi di personaggi poco o non altrimenti noti. Può essere opportuno osservare che nel Voyage il Montaigne menziona i cardinali Farnese, Carafa, Medici e altri ma senza offrire indizi di personale familiarità con essi ${ }^{118}$.

\section{Tra comune laico e potere pontificio, la civitas. Osservazioni Conclusive}

Pare a questo punto opportuno meglio contestualizzare queste riflessioni, in particolare per la dialettica più volte richiamata tra municipalità e Papato, che ha una storia e una evoluzione non sempre nel segno della continuità, e che solo un secolo prima era degenerata in contrasto e in scontro violento; e a tale proposito si può ricordare la congiura di Stefano Porcari contro il pontefice Nicolò V $(1453)^{119}$. A metà secolo XV, il Papato tende al controllo pieno degli organismi comunali i quali rivendicano formalmente sovranità e autogoverno. Sul piano istituzionale, della nomenclatura e delle funzioni civiche, essi si rapportano in modalità simbiotiche ai mitologemi del passato della respu-

115 Per Agustín ed Egio nel contesto del circolo romano, vd. Crawford, Agustín, 1-4 e ID., "Egio and the Development of Greek Epigraphy", ibid., 133-154, part. 136-7; R. Cooper, "Epigraphical Research in Rome in the Mid-Sixteenth Century: The Papers of Antonio Agustín and Jean Matal", in Crawford, Agustín, p. 95-7, 101-2, 103, 107, 110-1; Carbonell, Correspondance in Crawford, Agustín, p. 117-9, 123-126.

116 1510-1583. Nel 1549 Pirro Ligorio a Roma è al servizio di Ippolito II d'Este, pure citato nella lista di Goltzius, per dipingere la decorazione nel suo palazzo a Monte Giordano. L'attività presso l'Estense in Quirinale dura fino al 1557 quando il cardinale si trasferisce e Ligorio passa alle dirette dipendenze di Paolo IV e poi di Pio IV, per il quale completa la Casina di Pio IV nei Giardini Vaticani. Nel 1565 è architetto a San Pietro al posto di Michelangelo, ma nel 1565, dopo l'elezione di Pio V, Ligorio è accusato di malversazione e costretto a partire, dopo aver lavorato a Villa d'Este dietro richiesta del cardinale Ippolito e del cardinale Alessandro Farnese, anch'egli menzionato nella lista goltziana. Quindi si trasferisce presso gli Estensi a Ferrara, senza più rientrare a Roma. Vd. C.D. Coffin, Pirro Ligorio. The Renaissance Artist, Architect, and Antiquarian, Penn State Press, 2003.

117 1507-66. Cultore di antichità e traduttore di classici, drammaturgo e poeta, legato ai Farnese e in particolare a servizio del cardinale Alessandro Farnese come suo segretario.

118 Journal de Voyage, cap. 72: il giorno di Natale a San Pietro il Montaigne assiste alla messa tra il pubblico, pur da una postazione privilegiata, e i cardinali Farnese e Carafa officiano con il Papa.

119 Su cui vd. A. Modigliani , "La famiglia Porcari tra memorie repubblicane e curialismo", in Un pontificato e una città. Sisto IV (1471-1484), Atti del Convegno (Roma, 3-7 dicembre 1984), Roma, 1986, 317-353; EAD., L'eredità di Cola di Rienzo. Gli statuti del Comune di Popolo e la riforma di Paolo II, 2, in A. Rehberg, A. Modigliani, Cola di Rienzo e il Comune di Roma, Roma, 2004, p. 118; A. D’EliA, "Stefano Porcari's Conspiracy against Pope Nicholas V in 1453 and Republican Culture in Papal Rome", Journal of the History of Ideas, 68 (2007), p. 207-231. 
blica, a garanzia di un'autonomia formalmente scevra da ingerenze «imperiali» di poteri esterni. In tale clima di tensione era accaduto che per l'elezione del Senatore e degli altri magistrati capitolini, spettante per statuto alle alte rappresentanze del Comune popolare ${ }^{120}$, il Pontefice non venisse neppure interpellato. Nel 1469 papa Paolo II decreta un'importante riforma degli Statuti cittadini, che prevede tutta una serie di censure, emendamenti e correzioni agli Statuti popolari precedenti, nella direzione del potenziamento formale dell'autorità pontificia sulle istituzioni municipali. Le motivazioni ufficiali dell'intervento sono dichiarate nella bolla pontificia di conferma ${ }^{121}$ : stante la varietas temporum, Roma in quanto città-simbolo rappresenta la sede centrale dell'impero romano e la madre del diritto dei popoli, il luogo sacro cristiano per i martiri e i santi e soprattutto la sede apostolica prescelta; dunque il Pontefice, la cui sovranità è di investitura divina, ha il dovere di occuparsi di urbs, cives e populus come di propri figli paterna caritate; e dal momento che gli Statuti popolari, emanati troppo tempo addietro, per buona parte non garantiscono libertà alla Chiesa (statuta [...] nonnulla ecclesiastice libertati contrarie [...]) e dunque sono inadatti ai tempi, si è ora autorizzati a procedere con innovazioni idonee a restaurare il controllo pontificio sulla città: pontifex - imperator secondo il modello costantiniano nelle cerimonie ufficiali, di fatto Urbis dominus, il Papa promuove riformulazione e gerarchizzazione delle istituzioni municipali, nel segno della formale sottomissione al dominio pontificio. Le grandi famiglie dei baroni, che continuano a costituire de facto un ceto forte, ricercato nei rapporti e nelle relazioni anche molto al di là dei confini urbici, estromesse de iure dalla municipalità romana popolare e anti-magnatizia dal XIV secolo, per il Pontefice non costituiscono alleato affidabile, pur ambito. Dunque il Papato, di fatto regime assolutista ma costretto a confrontarsi con gli altri poteri dell'Urbe, può presentarsi anche come depositario di valori e istanze popolari, avvalendosi quando possibile dell'alleanza ora imposta ora condivisa con la nuova nobilitas ${ }^{122}$ sempre nel segno della continuità con la dimensione ideale dell'antico, pur con prevalente riferimento alla Roma augustea e imperiale ${ }^{123}$ più che repubblicana. Nel tentativo di ricercare l'origine dei titoli di questa nobilitas, si è osservato che le famiglie indicate nelle liste cinquecentesche come le più antiche non sono per la maggioranza altrimenti individuabili ancora a inizio XV secolo. È una nobiltà recente quella che nel corso del Cinquecento, e particolarmente dopo il 1527, si ricostituisce aprendosi a graduali acquisizioni «esterne», con politiche esogamiche e

120 Ossia, tra gli altri, gli Esecutori di giustizia, i Conservatori, i Consoli delle Arti, i Caporioni: Modigliani, Cola di Rienzo, p. 118-9.

121 Testo e discussione in Modigliani, Cola di Rienzo, p. 129-131.

122 L'oligarchia di censo costituita dalla nobiltà minore, ex popolari, mercanti, banchieri, pescivendoli etc.

123 Per titoli e simbologia: il Pontefice vivifica il mito del pater patriae, del restaurator Urbis, del principe pius e iustus, così da legittimarsi nell'autorità politico-religiosa e nel potere temporale a prescindere dalla «donazione di Costantino». 
con gli strumenti della scelta sostitutiva degli eredi ${ }^{124}$ e dell'assegnazione della civitas, di poi stabilizzandosi e arroccandosi ufficialmente in ceto oligarchico. Anche l'esame della documentazione sul Consilium Urbis conduce a conclusioni similari: nella prima metà del Cinquecento la quantità degli aventi diritto al voto si amplia per il numero dei forestieri che, divenuti cives privilegio, possono esercitare gli stessi diritti politici attivi dei cives origine ${ }^{125}$ ovvero, meglio, di parte di essi. Il punto d'arrivo del lungo processo, il «libro d'oro» della nobiltà romana con la lista ufficiale dei nominativi delle famiglie, verrà reso pubblico piuttosto tardi, nel 1746, con la bolla Urbem Romam di papa Benedetto XIV ${ }^{126}$, che ne legittima l'assetto. Ma già nel 1590 si considera terminata la fase di integrazione delle famiglie non romane pervenute a Roma e assimilate alla cittadinanza e al patriziato nel corso del secolo.

La concessione della cittadinanza romana con privilegio, pur nella dialettica con il potere pontificio, resta appannaggio dell'aristocrazia municipale. Il secolo XVI registra il culmine degli arrivi di stranieri a Roma con corrispondente accrescimento nel numero di richieste di accesso alla civitas, una eventuale disciplina nelle assegnazioni non si evince sul piano normativo ${ }^{127}$. Nelle minute con le registrazioni non si osservano sezioni separate o una qualche forma di gerarchizzazione delle assegnazioni, ad esempio in rapporto alla rilevanza sociale dei beneficiari. E tuttavia sono rilevabili divergenze e distinzioni, nella tipologia di stesura, nei formulari, nello stile e infine nel contenuto stesso delle trascrizioni degli scribi. Poco più di una registrazione, con le espressioni di rito ridotte al minimo o quasi del tutto abbreviate e solo la menzione dei Consoli, è l'aspetto della maggioranza dei privilegia civilitatis con finalità per lo più pratica, destinati a personaggi di minor rilievo o del tutto comuni; e se tali, a essi non si demandava con la cittadinanza la gestione degli incarichi municipali ovvero di fatto l'ingresso nella compagine della locale nobiltà. Inoltre i requisiti previsti precludono agli stranieri non possidenti, poveri o nullatenenti l'accesso alla civitas, con una sorta di pre-selezione che consente domanda, e integrazione ufficiale, ai soli residenti e possidenti; inoltre, per impulso dell'oligarchia dominante, vengono ad attivarsi ulteriori meccanismi dirimenti che agiscono da filtro, in qualche modo distinguendo dall'interno i modelli e limitando la fruizione dei diritti. La cittadinanza onoraria non parrebbe sottoposta a limitazioni:

124 Si tratta del fedecommesso per la conservatio bonorum e la conservatio dignitatum familiarum, strumento giuridico per evitare l'estinzione del casato: in mancanza di eredi diretti si trasmettevano i beni e le insegne di nobiltà a esponenti scelti di altre famiglie.

125 M. FranCESChini, "Dal consiglio pubblico e segreto alla congregazione economica: la crisi delle istituzioni comunali tra xvi e XVII secolo", in Il Comune di Roma, 337-362, part. 355-6.

126 MorI, Tot reges, EAD, "Nascita, evoluzione e regolamentazione pontificia del titolo di 'nobile romano'”, in L'identità genealogica e araldica. Fonti, metodologie, interdisciplinarità, prospettive, Atti del XIII Congresso internazionale di scienze genealogica e araldica (Torino, 21-26 settembre 1998), Roma 2000, 875-895, part. 875 ss.

127 Mori, Nobile romano, p. 877-878, 880-882. 
per i viri illustri vel clari non romani di nobile stirpe e/o di rinomato valore personale non dovrebbero osservarsi disparità né limitazioni nei diritti; ma la minuta del decreto per Montaigne che parrebbe accostarne il testo, dal formulario comune, ai senatoconsulti consueti per i comuni aspiranti cittadini, spesso già residenti, rappresenta indizio di distinzione. Può essere allora qui opportuno ricordare, con la Pavan, che solo alcuni cittadini onorari a Roma godevano del diritto di battere moneta ${ }^{128}$.

Preclusioni all'esercizio delle magistrature capitoline sono decretate dagli Statuti del 1519, e ribadite quindi negli Statuti del 1580 promulgati da Gregorio XIII ${ }^{129}$, nei quali con dettagliata elencazione dei requisiti si rende più difficoltoso l'accesso alle cariche municipali e a qualsiasi ufficio della Camera Capitolina, contribuendo alla chiara distinzione dei nobili dagli altri ceti ${ }^{130}$. Tenendo conto del fatto che gli Statuti non rappresentano punti di partenza, ma documentano tendenze e fenomeni in atto che infine si cristallizzano nella norma, si può presumere che tali disparità rappresentassero realtà antecedenti. Ai Conservatori si delega qui con evidenza la concessione di entrambe le modalità di cittadinanza, ai residenti proprietari di immobili e "honoris gratia illustribus vel claris viris postulantibus et cum assensu omnium vel maioris partis ipsorum ${ }^{131}$ "; e a questi, spesso anche prelati nonostante l'espresso divieto degli Statuti, è consentito accedere al Senato e godere degli onori e privilegi propri dei cives origine ovvero essere accolti senza remore nella oligarchia dominante.

Entrambi i nostri decreti rientrano in questa seconda tipologia. Per Hubertus Goltzius (1567) l'adesione è piena. Si è osservato come la formula impiegata per elencare i suoi diritti di civis sia assai circostanziata: [...] NOS (CONSULES) [...] FREQUENTIque senatu assistentibus, Hubertus Goltzius Herbipolitam Venlonianum, bene de ROMANA REPUBl. MERITUM, [...] CIVEM ROMANUM EX S.C. CREAVIMUS, CREATUM IN SENATUM VENIRE, MAGISTRATUS GERERE, IUS FERENDI SUFFRAGII HABERE, SACERDOTIA OBTINERE, BONA EIUS LIBERA ATQUE IMMUNIA ESSE, CETERISQUE ROMANORUM CIVIUM HONORIBUS ATQUE PRIVILEGIIS HONESTARI QUIBUS ILLI FRUUNTUR QUI CIVES ROMANI NATI AUT IURE OPTIMO FACTI SUNT. In quanto civis, Goltzius può non solo accedere al senato ma anche alle altre magistrature capitoline in rapporto alle quali può eleggere ed essere eletto, ottenere incarichi ecclesiastici, avere libertà e immunità di beni, conseguire gli altri onori e privilegi dei cittadini originari o di pieno diritto. In mancanza di un sacerdozio

128 Pavan, Cives, p. 39.

129 Ugo Buoncompagni, 1573-1585. È il papa delle grandi riforme: la ristrutturazione edilizia nell'Urbe per il giubileo del 1575; il sostegno a forme di proselitismo ed evangelizzazione, anche per contrastare l'azione della Riforma; la promulgazione degli Statuti, a rafforzamento del potere ecclesiastico. A questo papa si deve la realizzazione della Galleria delle Carte Geografiche in Vaticano per opera del cosmografo Egnazio Danti, con la rappresentazione dell'Italia del XVI secolo ripartita sulle grandi pareti affrescate. Importanti le valenze simboliche di tale cartografia, con la proiezione dello Stato Pontificio, sull'intera Italia.

130 Mori, Nobile romano, p. 878-880.

131 Mori, Nobile romano, p. 879-881, da cui è tratta la citazione (n. 12). 
municipale, la menzione dei sacerdotia da obtinere rappresenta in forma classicheggiante una ulteriore riprova della benevolenza degli ambienti clericali di potere, disponibili, parrebbe, a conferire al Goltzius anche incarichi ecclesiastici; peraltro un tale intervento in un decreto di civitas, dal dichiarato carattere laico, unitamente agli altri elementi valutati e indizianti l'operatività del potere religioso, assume un suo significato. Adulato ed esaltato dalla corte pontificia e dal Comune laico, in una fase in cui il Papa mostra il controllo del Consiglio, Goltzius mantiene a Roma relazioni elevate con il Pontefice stesso, i cardinali, i vescovi, gli intellettuali e gli artisti attivi nell' entourage ecclesiastico. Anche grazie alle mediazioni di Abraham Ortelius e soprattutto di Marc Laurin, egli è molto apprezzato dagli Asburgo di Spagna e d'Austria, oltre che dai circoli e dai personaggi di potere e di cultura più potenti d'Europa; basti pensare, solo a titolo di esempio, alle relazioni con i banchieri Függer di Augusta e con gli Schetz di Anversa ${ }^{132}$.

Per il Montaigne (1581), la formula relativa ai suoi diritti di civis diverge perché scarsamente o per nulla circostanziata: IPSUM POSTEROSQUe in RoMANAM Civitatem ADSCRIBI ORNARIQUE OMNIBUS ET PRAEMIIS ET HONORIBUS QUIBUS ILLI FRUUNTUR QUI Cives Patricilque Romani nati aut jure optimo facti sunt. Egli può trasmettere la cittadinanza in forma ereditaria e conseguire tutti i privilegi e gli onori dei cittadini e nobili romani originari o di pieno diritto, senza alcuna specificazione o dettaglio; non v'è cenno ai doveri. Tale dicitura generica se non esprime restrizioni nella gestione effettiva delle prerogative della civitas, non elencandoli non rende espliciti i diritti nel loro possibile esercizio; né è sufficiente essere civis Romanus per condividere e praticare in forma unitaria e omogenea tutte le prerogative del ceto. La cittadinanza, in particolare se onoraria, dovrebbe costituire uno strumento di integrazione indifferenziata sia in rapporto al corpo sociale nella sua interezza che all'oligarchia dominante; e tuttavia così non è. La genericità nello stile e nei contenuti, la facies dimessa della stesura del decreto del pur nobile Montaigne, nella sua analogia formale con i privilegia consueti per stranieri altrimenti ignoti, a confronto con il magniloquente e circostanziato testo per Goltzius che tutto dettaglia e consente, ne sono indizio. Dunque pur ponendosi tra le contrapposte possibilità del distinguere sulla base di limitazioni o di privilegi e dell' uniformare i cives alla medesima normativa giuridica ${ }^{133}$, lo strumento della cittadinanza non si presenta unitario nella formulazione e nell'esercizio.

Si può però legittimamente dubitare del reale interesse del Montaigne per l'aspetto «operativo» della Romana Civitas, tanto ambita dalla sua umana vanità. A Roma non destano il suo interesse i resti monumentali del passato, scarsamente rappresentativi dell'antica grandezza ${ }^{134}$; piuttosto lo attrae la storia stessa dell'Urbs, con le grandi

132 Ancora dalla lista del C. Iulius Caesar, Antverpiae: Gaspar, Melchior, Balthasar, Chuonradus Schetz; Augusta Vindelicorum (secondo viaggio): Ioannes Iacobus Függer, Raimundus Függer.

133 Opportune riflessioni su tale tematica in FECI, Migrazioni, p. 6-8.

134 Journal de Voyage, cap. 72. 
personalità che ne hanno scandito momenti irripetibili, in una sorta di visione relativistica del divenire storico e dell'uomo in cui ogni elemento ha valore in sé e non può costituire modello o, con altri, sistema di riferimento ${ }^{135}$. L'idea di Roma antiqua non rivela esemplarità di per sé, mentre l'esperienza romana è costantemente confrontata dal Montaigne con la propria sensibilità critica, con lo scetticismo del pensatore libero e perciò anche consapevole dei vincoli dell'esistenza ${ }^{136}$. La tanto ricercata Romana civitas deve aver avuto per lui, come per altri intellettuali di questi anni, non più che il valore di una personale adesione all'ideale cosmopolita e di riferimento per i popoli che Roma, l'Alma Urbs, nel Cinquecento universalmente esprimeva ${ }^{137}$.

\begin{tabular}{l} 
Maria Luisa NAPOLITANO \\
\hline corso Umberto, 72 \\
80042 - Boscotrecase (Na) \\
Italie \\
marialuisa.napolitano@unina.it
\end{tabular}

135 A. Giardina, A. Vauchez, Il mito, p. 88.

136 N. PANiCHI, I vincoli del disinganno, part. p. 265-270.

137 Journal de Voyage, cap. 74 (Roma 15 marzo-3 aprile 1581): "Roma è la città più cosmopolita del mondo, in cui il fatto di essere stranieri e le differenze di nazionalità contano meno; poiché per sua natura contiene forestieri dappertutto e ognuno vi si trova come a casa propria.» Si confronti l'opinione di Erasmo da Rotterdam, lettera a Jacopo Sadoleto, Basilea, 1 ottobre 1528, riportata in F. Gregorovius, Storia della città di Roma nel Medioevo, VIII, Milano, 1988 [1859-72], p. 610: "La disgrazia di Roma [il sacco di Roma del 1527] fu disgrazia di tutte le nazioni poiché quella città non era soltanto la rocca della religione cristiana, la nutrice degli eletti ingegni, il tranquillissimo asilo delle Muse, ma anche la madre comune di tutti i popoli. E veramente quale mai fu l'uomo, per quanto pur nato in terra straniera, che quella città non accogliesse nel suo placito seno, che non ve lo riscaldasse, che non lo educasse? Chi pareva di essere ivi straniero, fosse pur venuto dall'estremo angolo del mondo? [...].» Gli osservatori dei secoli XVI-XVII riconoscono il carattere cosmopolita dell'Urbe, in cui per intellettuali e uomini di cultura stranieri non è difficile integrarsi, soprattutto mediante la cittadinanza (A. Menniti Ippolito, I due «senati», part. p. 457). 